\title{
Star pentagon and many stable choreographic solutions of the Newtonian 4-body problem
}

\author{
Tiancheng Ouyang \\ Department of Mathematics, Brigham Young University \\ Provo, Utah 84602, USA \\ Email: ouyang@math.byu.edu \\ Zhifu Xie \\ Department of Mathematics and Computer Science \\ Virginia State University \\ Petersburg, Virginia 23806, USA \\ Email: zxie@vsu.edu
}

\begin{abstract}
In this paper, we give a rigorous proof of the existence of infinitely many simple choreographic solutions in the classical Newtonian 4-body problem. These orbits are discovered by a variational method with structural prescribed boundary conditions (SPBC). This method provides an initial path that is obtained by minimizing the Lagrangian action functional over the SPBC. We prove that the initial path can be extended to a periodic or quasiperiodic solution. With computer-assistance, a family of choreographic orbits of this type is shown to be linearly stable. Among the many linearly stable simple choreographic orbits, the most extraordinary one is the stable star pentagon choreographic solution (see figure 1). We also prove the existence of infinitely many double choreographic periodic solutions, infinitely many non-choreographic periodic solutions and uncountably many quasi-periodic solutions. Each type of periodic solutions has many stable solutions and possibly infinitely many stable solutions. Our results with SPBC largely complement the current results by minimizing the action on a loop space.
\end{abstract}

Key word: Variational Method, Choreographic Periodic Solutions, Variational Method with Structural Prescribed Boundary Conditions (SPBC), Stability, Central Configurations, $n$-body Problem.

AMS classification number: 37N05, 70F10,70H12, 70F15, 37N30, 70H05,

\section{Introduction}

Periodic solutions to the Newtonian $n$-body problem have been extensively studied for centuries. Variational method has been applied to obtain solutions for the $n$-body problem more than one hundred years since Poincaré [28] in 1896. In the past decade, the existence of many new interesting periodic orbits are proved by using variational method for the n-body problem. Most of them are found by minimizing the Lagrangian action on a symmetric loop space with some topological constraints (for example, see $[2,3,11,15,16,17,35,36]$ ).

(C) 2015. This manuscript version is made available under the Elsevier user license http://www.elsevier.com/open-access/userlicense/1.0/ 


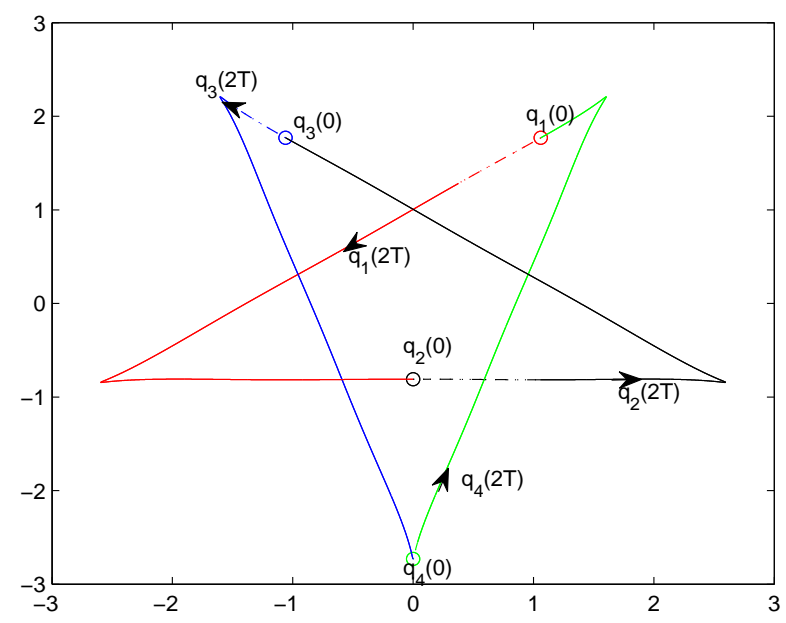

Figure 1: The first 40-th of the star pentagon, traveling from an isosceles triangle $q_{i}(0)$ with one in the axis of its symmetry to a trapezoid, and then back to another isosceles triangle $q_{i}(2 T)$. The entire star pentagon can be assembled by reflection, permutation, and rotation of the initial pieces (dashdotted). Initial conditions are: $q_{1}=(1.0598738926379,1.7699901770118)$, $\dot{q}_{1}=(-0.55391384867197,-0.39895079845794), \quad q_{2}=(0,-0.80951135793043), \quad \dot{q}_{2}=$ $(1.0936551555351,0), q_{3}=(-1.0598738926379,1.7699901770118), \dot{q}_{3}=(-0.55391558212647$, $0.39895379682134), q_{4}=(0,-2.7304689960932), \dot{q}_{4}=(0.01417427526245,0), m_{1}=m_{2}=$ $m_{3}=m_{4}=1, T=1$, period $=40$.

Following the notions in $[2,9]$, a simple choreographic solution (for short, choreographic solution) is a periodic solution that all bodies chase one another along a single closed orbit. If the orbit of a periodic solution consists of two closed curves, then it is called a doublechoreographic solution. If the orbit of a periodic solution consists of different closed curves, it is called a non-choreographic solution to emphasize that each closed curve is the trajectory of exact one body. Many relative equilibria give rise to simple choreographic solutions and they are called trivial choreographic solutions (circular motions). The first remarkable nontrivial choreographic solution - the figure eight of the three body problem was discovered numerically by Moore (1993 [24]) and it was proved by the variational method by Chenciner and Montgomery (2000, [8]). Zhang and Zhou ([38]) offer a new shorter proof of the existence of the figure-eight choreography solution. Many expertises attempt to study choreographic solutions and a large number of simple choreographic solutions have been discovered numerically but very few of them have rigorous existence proofs. More results can be found in $[1,4,7,9,10$, $12,13,14]$ and the reference therein.

Figure eight is a remarkably non-trivial simple choreographic solution, but more importantly, it is stable and the stability was proved in ([20, 21, 30]). Most important to astronomy are stable periodic solutions which means that there is some chance that such periodic solutions might actually be seen in some stellar system. To the best knowledge of the authors, all of the above known simple choreographic solutions are unstable except the figure eight. It seems very hard to find a stable simple choreographic solution (C. Simó [32] and R. Vanderbei [37]). However, many simple choreographic orbits in this paper are linearly stable.

In this paper, instead of considering the whole path in some loop space with some topological or geometric symmetric constrains, we consider the boundary value problem with ap- 
propriate boundary configurations which are given by two $n \times d$ matrices, Qstart and Qend. For instance in the following theorem 1.1, $q(0)=$ Qstart and $q(T)=$ Qend are two $4 \times 2$ matrices that Qstart forms an isosceles triangle with one in the axis of the triangle and Qend forms a trapezoid. A two-step minimizing process is utilized to find some appropriate pieces of orbits which are proven to be assembled out to a periodic solution (or a quasi-periodic solution). To emphasize this process with boundary conditions, it is called the variational method with Structural Prescribed Boundary Conditions (SPBC). This idea of SPBC had been reported by one of the authors, Tiancheng Ouyang in the HAMSYS conference, March 2001 (see Chenciner's Remark in [6]). Some preliminary results were included in the paper [13] which was submitted in 2003 and was finally published in 2012. The method works for general $n$-body problem without any constraints on masses or symmetries.

Using the proposed variational method with SPBC, we give rigorous existence proofs of infinitely many simple choreographic solutions in the Newtonian 4-body problem with equal masses. Significantly, a family of choreographic orbits of this type are all linearly stable by numerical analysis. Among the many stable simple choreographic orbits, the most extraordinary one is the stable star pentagon choreographic solution (see Figure 1). The star pentagon is assembled out of four pieces of curves which are obtained by minimizing the Lagrangian action functional over the SPBC. We also prove the existence of infinitely many double choreographic periodic solutions, infinitely many non-choreographic periodic solutions and uncountably many quasi-periodic solutions. Each type of periodic solutions have many stable solutions and possibly infinitely many stable solutions.

Theorem 1.1. Consider the Newtonian 4-body problem with four equal masses. For any fixed $T>0$, and $\theta=\frac{2 \pi}{5}$, let the structural prescribed boundary conditions be two fixed boundary configurations $q(0)=\left(\begin{array}{cc}a_{1} & a_{2} \\ 0 & -a_{3} \\ -a_{1} & a_{2} \\ 0 & -2 a_{2}+a_{3}\end{array}\right)$ and $q(T)=\left(\begin{array}{rr}-a_{5} & a_{4} \\ a_{5} & a_{4} \\ -a_{6} & -a_{4} \\ a_{6} & -a_{4}\end{array}\right) R(\theta)$, where $\vec{a}=$ $\left(a_{1}, a_{2}, \cdots, a_{6}\right) \in \mathbf{R}^{6}$, and the rotation matrix $R(\theta)=\left(\begin{array}{cc}\cos (\theta) & -\sin (\theta) \\ \sin (\theta) & \cos (\theta)\end{array}\right)$. Then there exists an $\vec{a}_{0} \in \mathbf{R}^{6}$ such that a minimizing path $q^{*}(t)=\left(q_{1}^{*}(t), q_{2}^{*}(t), q_{3}^{*}(t), q_{4}^{*}(t)\right)$ on $[0, T]$ connecting $q(0)$ and $q(T)$ can be extended to a periodic solution $q(t)$ of the Newton's equation. It is proved that the periodic solution $q(t)$ (see Figure 1) has minimum period $\mathcal{T}=40 T$ with the following properties:

1. (Noncollision) $q_{i}(t) \neq q_{j}(t)$ for any $t$ and $i \neq j$.

2. (Choreographic) $q_{2}(t)=q_{1}(t+10 T), q_{3}(t)=q_{1}(t+20 T), q_{4}(t)=q_{1}(t+30 T)$, and $q_{1}(t)=q_{1}(t+40 T)$.

3. $\left(\right.$ Symmetry) $q_{1}(-t)=q_{3}(t) B, q_{2}(-t)=q_{2}(t) B, q_{3}(-t)=q_{1}(t) B$, and $q_{4}(-t)=q_{4}(t) B$, where $B=\left(\begin{array}{cc}-1 & 0 \\ 0 & 1\end{array}\right)$ is the reflection about $y$-axis, i.e. $q_{j}(t) B=\left(-q_{j 1}(t), q_{j 2}(t)\right)$.

4. (Geometric Transition) $\left\{q_{i}(2 k T)\right\}$ are vertices of an isosceles triangle with an interior point on the axis of the isosceles triangle. $\left\{q_{i}((2 k+1) T)\right\}$ are vertices of a trapezoid.

5. (Stability) $q(t)$ is a linearly stable star pentagon choreographic solution.

Remark 1.2. Numerically, if $T=1$ and $\theta=\frac{2 \pi}{5}$, then $\vec{a}_{0}=[1.0598738926379,1.7699901770118$, $0.80951135793043,0.75377929101531,1.1034410399611,2.440248251576]$ and the initial conditions are given in Figure 1. The star pentagon forms by assembling out the initial four pieces 
of curves starting from $q(0)$ to $q(T)$ (dashdotted line in Figure 1). The extension to the full star pentagon is done by reflection, permutation and rotation as in equation (11). The proof of the existence is rigorous but the proof of the stability is computer-assisted. During the revision process of this paper, the work of R. Broucke [5] came to our attention. Broucke had numerically searched the shape of the star pentagon solution in 2004 but no initial conditions are provided and he did not mention whether the star pentagon solution is stable.

Our main theorem for $\theta=\frac{2 \pi}{5}$ can also be extended for some other $\theta$.

Theorem 1.3. There exist $\theta_{0}$ and $\theta_{1}$ satisfying $\theta_{0}<\frac{\pi}{2}<\theta_{1}$ such that for any $\theta \in\left(\theta_{0}, \theta_{1}\right)$ and $\theta \neq \frac{\pi}{2}$, there exists at least one $\vec{a}_{0} \in \mathbf{R}^{6}$ such that the minimizing path $q^{*}(t)$ connecting $q(0)$ and $q(T)$ can be extended to a non-circular classical Newtonian solution by assembling out the initial pieces. If $\theta$ is not commensurable with $\pi$, the extension is a quasi-periodic solution. If $\theta$ is commensurable with $\pi$, the extension is a periodic solution. Among the periodic solutions, there are infinitely many choreographic periodic solutions and many of them are linearly stable. See Figure 5 to Figure 12 in appendix $C$.

Remark 1.4. The value of the action of the minimizing solution $q^{*}(t)$ is smaller than the action of the corresponding circular motion for $\theta \in\left(\theta_{0}, \theta_{1}\right)$. Numerically, $1.1938<\theta_{0}<1.2252$ or equivalently $0.38 \pi<\theta_{0}<0.39 \pi$, and $1.7279<\theta_{1}<1.7593$ or equivalently $0.55 \pi<\theta_{1}<0.56 \pi$. There also exist local minimizers which have higher actions than their circular solutions. By using canonical transformation, we eliminate the trivial +1 multipliers and we compute the eigenvalues of its monodromy matrix to prove that the simple choreographic solutions are linearly stable in the reduced space for $\theta=\frac{P}{2 P+1} \pi, P=2,3,4, \cdots, 15$. The non-choreographic solutions are linearly stable for $\theta=\frac{2 P-1}{4 P}, P=3,4, \cdots, 8$. The double choreographic solutions $q(t)$ are also linearly stable for $\theta=\frac{2 P-1}{4 P+2}, P=5,6,7$. From our calculation and numerical simulation program, periodic solutions with lower actions for $\theta \in\left(\theta_{0}, \theta_{1}\right)$ seem more likely stable. Periodic solutions for $\theta$ out of $\left(\theta_{0}, \theta_{1}\right)$ are more likely unstable. For example, we check that periodic solutions are linearly unstable for $\theta=\frac{1}{4} \pi, \frac{1}{3} \pi, \frac{3}{8} \pi, \frac{3}{10} \pi, \frac{4}{11} \pi, \frac{5}{14} \pi$ and so on. Our theorem 5.1 and numerical computation supports the following conjecture. To prove the conjecture, some new techniques may be involved such as index theory (see [19], [20], [22], [25]) but it is out of the scope of the current paper.

Conjecture: The non-circular periodic solutions in theorem 1.3 are all linearly stable for $\theta \in\left(\theta_{0}, \theta_{1}\right)$ and $\theta \neq \frac{\pi}{2}$ and there are infinitely many stable choreographic solutions.

Our paper is organized in the following manner. In section 2, we first briefly describe the variational method with structural prescribed boundary conditions for the special case $\theta=\frac{2 \pi}{5}$ and equal masses. The main theorem 1.1 is restated as theorem 2.3 to 2.6. Its proof is carried out in section 3 and the linear stability is studied in section 4 . Section 5 is devoted to the main theorem 1.3 with more details on the classification of periodic solutions with respect to the general rotational angle $\theta$. The calculations of the action of a path to generate a circular motion or the action of a test path are given in appendix A and appendix B respectively. Finally, some numerical simulations are given in appendix C.

\section{Settings and Restatements of Main Theorem 1.1}

Given $n$ bodies, let $m_{i}$ denote the mass and $q_{i}(t)$ denote the position in $\mathbf{R}^{d}, d \geq 2$ of body $i$ at time $t$ in $d$-dimensional space. The action functional is a mapping from the space of all 
trajectories $q_{1}(t), q_{2}(t), \cdots, q_{n}(t)$ into the reals. It is defined as the integral:

$$
\mathcal{A}(q(t))=\int_{0}^{T} \frac{1}{2} \sum_{i=1}^{n} m_{i}\left|\dot{q}_{i}(t)\right|^{2}+U(q(t)) d t,
$$

where $U$ is the Newtonian potential function

$$
U=\sum_{1 \leq i<j \leq n} \frac{m_{i} m_{j}}{\left|q_{i}-q_{j}\right|}
$$

Critical points of the action functional are trajectories that satisfy the equations of motion, i.e. Newton's equations:

$$
m_{i} \ddot{q}_{i}=\frac{\partial U}{\partial q_{i}}=\sum_{j=1, j \neq i}^{n} \frac{m_{i} m_{j}\left(q_{j}-q_{i}\right)}{\left|q_{j}-q_{i}\right|^{3}}, \quad 1 \leq i \leq n .
$$

Without loss of generality, we assume that the center of mass $\frac{1}{\sum_{i=1}^{n} m_{i}} \sum_{i=1}^{n} m_{i} q_{i}$ is always at the origin. Let the linear momentum be

$$
p_{i}=m_{i} \dot{q}_{i}
$$

Then the Hamiltonian governing the equations of motion is

$$
H(q, p)=\frac{1}{2} \sum_{i=1}^{n} \frac{\left|p_{i}\right|^{2}}{m_{i}}-U
$$

By the fundamental theorem of existence and uniqueness of differential equations, the second order nonlinear ODE system of Newton's equations has a unique solution for an appropriate initial conditions, i.e. the initial position vector $q(0)$ and the initial velocity vector $\dot{q}(0)$ determine its future motion. But the initial conditions are very local and it is very hard to directly determine the initial conditions to lead a periodic solution. Instead of considering the initial value problem, we consider the boundary value problem with appropriate prescribed boundary configurations on $q(0)$ and $q(T)$ so that the orbit connecting $q(0)$ and $q(T)$ can be extended to a periodic solution. Here we consider two appropriate boundary configurations Qstart $\in\left(\mathbf{R}^{d}\right)^{n}$ and Qend $\in\left(\mathbf{R}^{d}\right)^{n}$, and the path space

$$
\mathcal{P}(\text { Qstart }, \text { Qend }):=\left\{q(t) \in H^{1}\left([0, T],\left(\mathbf{R}^{d}\right)^{n}\right) \quad \mid \quad q(0)=\text { Qstart }, q(T)=\text { Qend }\right\} .
$$

A natural choice of the path space for the action functional $\mathcal{A}$ defined in (1) is the Sobolev space $H^{1}\left([0, T],\left(\mathbf{R}^{d}\right)^{n}\right)$, in which a critical point $q(t)$ of $\mathcal{A}$ is a classical solution of Newton's equation (2) on $[0, T]$, if and only if $q$ is collision free. The existence of minimizers in the Sobolev space is classic and standard. But the assertion of collision free for the boundary value problem is proved by Chenciner [6] and Marchal [23] in 2002.

Lemma 2.1. Given any Qstart $\in\left(\mathbf{R}^{d}\right)^{n}$ and Qend $\in\left(\mathbf{R}^{d}\right)^{n}$, minimizers of $\mathcal{A}$ on the space $\mathcal{P}($ Qstart, Qend $)$ are collision-free on the interval $(0, T)$.

Minimizers of $\mathcal{A}$ are classic solutions of the Newton's equation (2) and it is $C^{2}$ in $\mathcal{P}($ Qstart, Qend). Because linear momentum is an integral of motion, it is natural to assume that every path stays inside the configuration space $W$ :

$$
W:=\left\{q \in\left(\mathbf{R}^{d}\right)^{n} \quad \mid \sum_{i=1}^{n} m_{i} q_{i}=0\right\} .
$$


If Qstart and Qend are both in $W$, then the minimizers are always in $W$.

We will present the variational approach with the SPBC in the discovery of the stable star pentagon choreographic solution of the planar four-body problem with equal masses. The results can be extended for general $\theta$ in section 5. The method can be applied for unequal masses [26] and in three dimensional space [27]. In section 2 to $4, \theta=\frac{2 \pi}{5}, m_{1}=m_{2}=m_{3}=$ $m_{4}=1$ and dimension $d=2$. The essential part of this method is the choice of an appropriate SPBC in order to get a possible preassigned periodic orbit and in order to provide a proof of such existence.

\section{Structural Prescribed Boundary Conditions (SPBC):}

Let $\Gamma=\mathbf{R}^{6}$. The fixed Qstart and the fixed Qend are defined by Qstart $=$ $\left(\begin{array}{cc}a_{1} & a_{2} \\ 0 & -a_{3} \\ -a_{1} & a_{2} \\ 0 & -2 a_{2}+a_{3}\end{array}\right)$ and Qend $=\left(\begin{array}{rr}-a_{5} & a_{4} \\ a_{5} & a_{4} \\ -a_{6} & -a_{4} \\ a_{6} & -a_{4}\end{array}\right) R(\theta)$ for a given $\vec{a}=\left(a_{1}, a_{2}, \cdots, a_{6}\right) \in$

$\Gamma$. Then the set $S(\vec{a})$ of minimizers is defined by

$$
\begin{gathered}
S(\vec{a})=\left\{q(t)=\left(q_{1}, q_{2}, q_{3}, q_{4}\right)(t) \in C^{2}\left((0, T),\left(\mathbf{R}^{\mathbf{2}}\right)^{4}\right) \quad \mid \quad q(0)=\text { Qstart, } q(T)=\text { Qend },\right. \\
q(t) \text { is a minimizer of the action functional } \mathcal{A} \text { over } \mathcal{P}(Q \text { start }, \text { Qend })\} .
\end{gathered}
$$

So the configuration of the bodies changes from an isosceles triangle with one on the axis of symmetry of the triangle to a trapezoid for some positive $\vec{a}$.

In some case, $\Gamma$ can be a subspace or subset of $\mathbf{R}^{6}$. For any given $\vec{a} \in \Gamma$, the minimizers of $\mathcal{A}$ that connect $Q$ start and Qend are classical collision-free solutions in the interval $(0, T)$. Then the real value function $\tilde{\mathcal{A}}(\vec{a}): \Gamma \rightarrow \mathbf{R}$ is well defined by

$$
\tilde{\mathcal{A}}(\vec{a})=\int_{0}^{T} \frac{1}{2} \sum_{i=1}^{n} m_{i}\left|\dot{q}_{i}(t, \vec{a})\right|^{2}+U(q(t, \vec{a})) d t,
$$

where $q(t, \vec{a}) \in S(\vec{a})$ is a minimizer of the action functional $\mathcal{A}$ over $\mathcal{P}(Q$ start, Qend $)$ for the given $\vec{a} \in \Gamma$. If it is clear that $q(t, \vec{a})$ is a minimizer for the given $\vec{a}$ from context, we still use $q(t)$ for $q(t, \vec{a})$ for convenience. It is easy to know that $\tilde{\mathcal{A}}$ is lower semicontinuous on $\Gamma$. The existence of minimizers in the finite dimension space $\Gamma$ is due to the following proposition.

Proposition 2.2. For $\theta=\frac{2 \pi}{5}, \tilde{\mathcal{A}}(\vec{a}) \rightarrow+\infty$ if $|\vec{a}| \rightarrow+\infty$.

Proof. For any $\vec{a} \in \Gamma$,

$$
\tilde{\mathcal{A}}(\vec{a}) \geq \sum_{i=1}^{n} \int_{0}^{T} \frac{1}{2} m_{i}\left|\dot{q}_{i}(t, \vec{a})\right|^{2} d t \geq \sum_{i=1}^{n} \frac{1}{2} m_{i}\left|\int_{0}^{T} \dot{q}_{i}(t, \vec{a}) d t\right|^{2}=\sum_{i=1}^{n} \frac{1}{2} m_{i}\left|q_{i}(T)-q_{i}(0)\right|^{2} .
$$

By the triangle inequality $\left|q_{i}(T)-q_{i}(0)\right| \geq|| q_{i}(T)|-| q_{i}(0)||$ we have

$$
\tilde{\mathcal{A}}(\vec{a}) \geq \sum_{i=1}^{n} \frac{1}{2} m_{i}\left(\left|q_{i}(T)\right|-\left|q_{i}(0)\right|\right)^{2} .
$$

Let $\alpha_{i}$ be the angle between the vectors $q_{i}(0)$ and $q_{i}(T)$. If $0 \leq \alpha_{i}<\frac{\pi}{2}$, the length of the side $q_{i}(T)-q_{i}(0)$ is larger than the heights of the triangle

$$
\left|q_{i}(T)-q_{i}(0)\right| \geq \max \left\{\left|q_{i}(T)\right| \sin \left(\alpha_{i}\right),\left|q_{i}(0)\right| \sin \left(\alpha_{i}\right)\right\} .
$$



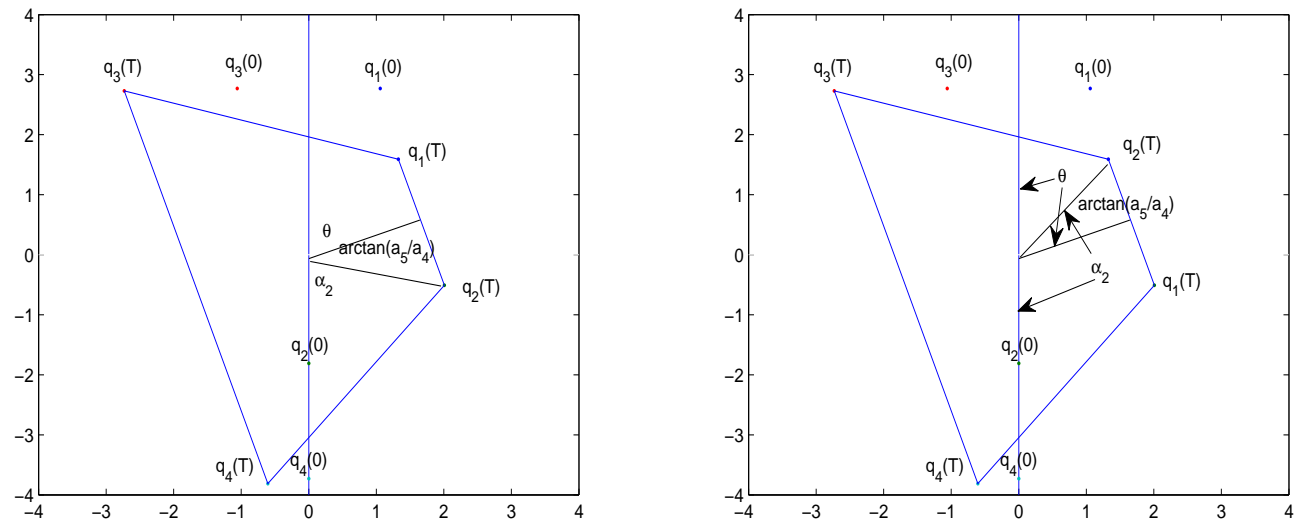

Figure 2: Left: If $\vec{a}>0,0<\alpha_{2}=\pi-\theta-\arctan \left(\frac{a_{5}}{a_{4}}\right)<\pi$. Right: $a_{5}<0$ and $a_{i}>0$ for $i \neq 5$, $\frac{\pi}{2}<\alpha_{2}=\pi-\theta+\arctan \left(\frac{a_{5}}{a_{4}}\right)$.

If $\frac{\pi}{2} \leq \alpha_{i} \leq \pi$, the length of the side $q_{i}(T)-q_{i}(0)$ is the longest side of the triangle

$$
\left|q_{i}(T)-q_{i}(0)\right| \geq \max \left\{\left|q_{i}(T)\right|,\left|q_{i}(0)\right|\right\} .
$$

By the inequality (7) and (8), we have

$$
\begin{aligned}
\tilde{\mathcal{A}}(\vec{a}) \geq & \sum_{0 \leq \alpha_{i}<\frac{\pi}{2}} \frac{1}{2} m_{i}\left|\max \left\{\left|q_{i}(T)\right| \sin \left(\alpha_{i}\right),\left|q_{i}(0)\right| \sin \left(\alpha_{i}\right)\right\}\right|^{2} \\
& +\sum_{\frac{\pi}{2} \leq \alpha_{j} \leq \pi} \frac{1}{2} m_{j}\left|\max \left\{\left|q_{j}(T)\right|,\left|q_{j}(0)\right|\right\}\right|^{2}
\end{aligned}
$$

If $\tilde{\mathcal{A}}(\vec{a})$ remains finite while $|\vec{a}|$ goes to infinity, then $q_{i}(0)$ and $q_{i}(T)$ must go to infinity for all $i$ by the structure of the SPBC and the inequality (6). Then $\sin \left(\alpha_{i}\right)$ goes to zero for all $i$ by the inequality (9), that is $\alpha_{i} \rightarrow 0$. But that $\alpha_{i} \rightarrow 0$ for all $i$ is impossible due to the structure of the SPBC. For example, if $a_{i} \geq 0$ for all $i$, the angle $\alpha_{2}$ would be $\pi-\theta-\arctan \left(\frac{a_{5}}{a_{4}}\right)$ (see Left in figure 2). Therefore $0<\frac{\pi}{2}-\theta=\frac{\pi}{10} \leq \alpha_{2}=\frac{3 \pi}{5} \leq \pi-\theta<\pi$ and $\sin \left(\alpha_{2}\right) \neq 0$ which prevent $\alpha_{2} \rightarrow 0$. Other cases can be easily obtained by the geometric structure of the SPBC and detail arguments are omitted.

Let $\vec{a}_{0}=\left(a_{10}, a_{20}, \cdots, a_{60}\right) \in \Gamma$ be a minimizer of $\tilde{\mathcal{A}}(\vec{a})$ over the space $\Gamma$ and the corresponding path $q^{*}(t)=q^{*}\left(t, \vec{a}_{0}\right) \in S\left(\vec{a}_{0}\right)$, i.e.

$$
\begin{aligned}
\tilde{\mathcal{A}}\left(\vec{a}_{0}\right) & =\min _{\vec{a} \in \Gamma} \tilde{\mathcal{A}}(\vec{a})=\min _{\vec{a} \in \Gamma}\left\{\inf _{q(t) \in \mathcal{P}\left(Q_{\text {start }}, Q_{\text {end }}\right)} \mathcal{A}(q(t))\right\} \\
& =\min _{\vec{a} \in \Gamma}\left\{\inf _{q(t) \in \mathcal{P}\left(Q_{\text {start }}, Q_{\text {end }}\right)} \int_{0}^{T} \frac{1}{2} \sum_{i=1}^{n} m_{i}\left|\dot{q}_{i}(t)\right|^{2}+U(q(t)) d t\right\} .
\end{aligned}
$$

Then the path $q^{*}$ is the solution we want and Theorem 1.1 can be proved immediately by the following theorems.

Theorem 2.3 (Noncollision). Let $\vec{a}_{0}$ be a minimizer of $\tilde{\mathcal{A}}(\vec{a})$ over the space $\Gamma$ and the corresponding path $q^{*}(t) \in S\left(\vec{a}_{0}\right)$. Then $q^{*}$ satisfying $S P B C$ is a classical solution of Newton's equation (2) in the whole interval $[0, T]$. 
Let $\mathbf{A}$ and $\mathbf{B}$ be two proper linear subspaces of $\left(\mathbf{R}^{2}\right)^{4}$ which are given as

$$
\mathbf{A}=\left\{\left(\begin{array}{cc}
a_{1} & a_{2} \\
0 & -a_{3} \\
-a_{1} & a_{2} \\
0 & -2 a_{2}+a_{3}
\end{array}\right) \in\left(\mathbf{R}^{2}\right)^{4} \mid\left(a_{1}, a_{2}, a_{3}\right) \in \mathbf{R}^{3}\right\}
$$

and

$$
\mathbf{B}=\left\{\left(\begin{array}{rr}
-a_{5} & a_{4} \\
a_{5} & a_{4} \\
-a_{6} & -a_{4} \\
a_{6} & -a_{4}
\end{array}\right) R(\theta) \in\left(\mathbf{R}^{2}\right)^{4} \mid\left(a_{4}, a_{5}, a_{6}\right) \in \mathbf{R}^{3}\right\}
$$

Let us consider the action functional $\mathcal{A}$ defined in (1) over the function space

$$
\mathcal{P}(\mathbf{A}, \mathbf{B}):=\left\{q \in H^{1}\left([0, T],\left(\mathbf{R}^{2}\right)^{4}\right) \mid q(0) \in \mathbf{A}, q(T) \in \mathbf{B}\right\} .
$$

It is easy to prove the lemma of equivalence below.

Lemma 2.4 (Equivalence). $\vec{a}_{0} \in \Gamma$ with corresponding path $q^{*} \in S\left(\vec{a}_{0}\right)$ satisfying $q^{*}(0)=$ Qstart and $q^{*}(T)=$ Qend is a minimizer of $\tilde{\mathcal{A}}(\vec{a})$ over the space $\Gamma$, if and only if, $q^{*}$ is a minimizer of $\mathcal{A}$ over the function space $\mathcal{P}(\mathbf{A}, \mathbf{B})$ with $q^{*}(0)=$ Qstart $\in \mathbf{A}$ and $q^{*}(T)=$ Qend $\in \mathbf{B}$.

Theorem 2.5 (Extension). For any local minimizer $\vec{a}_{0} \in \Gamma$ of $\tilde{\mathcal{A}}(\vec{a})$ over the space $\Gamma$, its corresponding path $q^{*}(t)=\left(q_{1}^{*}(t), q_{2}^{*}(t), q_{3}^{*}(t), q_{4}^{*}(t)\right)$ on $[0, T]$ can be extended to a periodic solution of the Newton's equation (2) by the reflection $B=\left(\begin{array}{cc}-1 & 0 \\ 0 & 1\end{array}\right)$, a permutation $\sigma$ and the rotation $R(\theta)$ as follows: $q(t)=q^{*}(t)$ on $[0, T]$,

$$
q(t)=\left(\left(q_{2}^{*}(2 T-t), q_{1}^{*}(2 T-t), q_{4}^{*}(2 T-t), q_{3}^{*}(2 T-t)\right) B\right) R(2 \theta) \quad \text { on } \quad(T, 2 T],
$$

and

$$
q(t)=\sigma^{k}(q(t-2 k T)) R(2 k \theta) \text { for } t \in(2 k T,(2 k+2) T] \text { and } k \in \mathbf{Z}^{+},
$$

where $\sigma=[2,3,4,1]$ is a permutation such that $\sigma(q(t-2 T))=\left(q_{2}(t-2 T), q_{3}(t-2 T), q_{4}(t-\right.$ $\left.2 T), q_{1}(t-2 T)\right)$. There exists a local minimizer $\vec{a}_{0}$ such that its corresponding path produces a star pentagon choreographic solution (see Figure 1) of the Newton's equation (2) with minimum period $40 T$.

Theorem 2.6. Star pentagon choreographic solution is linearly stable.

Remark 2.7. (1) These theorems assert that the initial pieces of orbits are extended to a periodic solution by assembling the pieces themselves. From the extension equation (11), it is easy to prove the properties in theorem 1.1. Note that, we do not assume that the solutions have symmetries and we do not impose symmetry constraints to prove the existence of solutions. Instead, we prove that the periodic or quasi-periodic solutions that are extended from our initial minimizing pathes satisfying SPBC have these symmetries.

(2) Proposition 2.2 is generally true for $\theta \in(0, \pi) \backslash\left\{\frac{\pi}{4}, \frac{\pi}{2}, \frac{3 \pi}{4}\right\}$. It can be proved by simply noticing that $\mathbf{A} \cap \mathbf{B}$ is empty for $\theta \in(0, \pi) \backslash\left\{\frac{\pi}{4}, \frac{\pi}{2}, \frac{3 \pi}{4}\right\}$. When $\theta \in\left\{\frac{\pi}{4}, \frac{\pi}{2}, \frac{3 \pi}{4}\right\}$, there exists a sequence $\vec{a}_{n}$ such that $\tilde{\mathcal{A}}\left(\vec{a}_{n}\right)$ remains finite while $\left|\vec{a}_{n}\right| \rightarrow \infty$ when $n \rightarrow \infty$. 


\section{Proof of Theorem 2.3 to 2.5}

The proof of theorem 2.3. If $\vec{a}_{0}$ is a minimizer of $\tilde{\mathcal{A}}(\vec{a})$ over the space $\Gamma$, we only need to prove that $Q \operatorname{start}\left(a_{10}, a_{20}, a_{30}\right)$ and $\operatorname{Qend}\left(a_{40}, a_{50}, a_{60}\right)$ have no collision. In fact, there are six cases corresponding to initial collision boundary. (1) $a_{10} \neq 0$ and $a_{20}=a_{30}$ binary collision $\left(m_{2}\right.$ and $m_{4}$ collide). (2) $a_{10}=0, a_{20} \neq-a_{30}$, and $a_{20} \neq \frac{1}{3} a_{30}$, binary collision ( $m_{1}$ and $m_{3}$ collide). (3) $a_{10}=0$, and $a_{20}=a_{30} \neq 0$ simultaneous binary collision. (4) $a_{10}=a_{20}=a_{30}=0$ total collision. (5) $a_{10}=0, a_{20}=-a_{30} \neq 0$ triple collision $\left(m_{1}, m_{2}\right.$, and $m_{3}$ collide). (6) $a_{10}=0, a_{20}=\frac{1}{3} a_{30} \neq 0$ triple collision $\left(m_{1}, m_{3}\right.$, and $m_{4}$ collide). There are five cases corresponding to ending collision boundary. (7) $a_{40} \neq 0, a_{50}=0$ and $a_{60} \neq 0$ binary collision ( $m_{1}$ and $m_{2}$ collide). (8) $a_{40} \neq 0, a_{50} \neq 0$ and $a_{60}=0$ binary collision ( $m_{3}$ and $m_{4}$ collide). (9) $a_{40} \neq 0, a_{50}=a_{60}=0$ simultaneous binary collision. (10) $a_{40}=a_{50}=a_{60}=0$ total collision. (11) $a_{40}=0, a_{50}=a_{60} \neq 0$ simultaneous binary collision.

Since $q$ has no collision in the open interval $(0, T)$, we will then analyze the motion during the closed time interval $[0, \epsilon]$ or $[\epsilon, T]$ and prove the existence of sufficiently small values of $\epsilon$ such that a local deformation has lower action and satisfy the SPBC. The contradiction proves that $q$ can not have this collision. Local deformation argument has appeared in a number of papers such as Marchal [23], Chenciner [6], Ferrario-Terracini [17], and Terracini-Venturelli [36] etc. Our proof follows the papers of Marchal [23] and Chenciner [6], but a few technique arguments are proved differently (especially the construction of deformation with SPBC). Here we only study the collisions at $t=0$ and similar arguments can be applied for collisions at $t=T$. By the nature of SPBC and the construction of the local deformation, we will prove it in two cases: collision with two bodies or collision with three or more bodies.

\section{CASE ONE: Collision with two bodies.}

Suppose that $q$ is a local minimizer of $\mathcal{A}$ satisfying the SPBC for $\vec{a}_{0}$. Let the collision subset $\mathcal{C}=\left\{\tau_{1}, \tau_{2}\right\} \subseteq\{1,2,3,4\}$. At time $t=0$, the bodies $m_{\tau_{1}}$ and $m_{\tau_{2}}$ start at the collision point $q_{\tau_{1}}(0)=q_{\tau_{2}}(0)$ while the other bodies are away. By the structural of SPBC, the collision set $\mathcal{C}$ must be either $\{1,3\}$ or $\{2,4\}$ which is corresponding to the binary collisions (1), (2) and (3) at $t=0$.

We will build the two following pathes $S_{2}$ (Kepler ejection orbits at the starting point) and $S_{3}$ (the deformation of $S_{2}$ ) with: (A) Exactly the same motion of all bodies in the interval $[\epsilon, T)$. (B) At the time interval $[0, \epsilon]$, the ejection orbits are replaced by a collision free orbits with boundary conditions satisfying SPBC. The corresponding actions will be $A_{1}=\mathcal{A}(q)$, $A_{2}=\mathcal{A}\left(S_{2}\right), A_{3}=\mathcal{A}\left(S_{3}\right)$. We want to prove that $A_{1}>A_{3}$ for sufficiently small time $\epsilon$. Since (A), the actions are different only in the time interval $[0, \epsilon]$.

First, consider the ejection orbits in the starting time interval $[0, \epsilon]$ in $S_{2}$. Let $r$ be the simple radial two-body motion leading from 0 to $r_{\epsilon}$ in the time interval $[0, \epsilon]$. By Sundman and Sperling's estimates near collisions [33, 34], there exists a positive constant $\gamma$ such that

$r(t)=\left(\gamma t^{\frac{2}{3}}\right) \vec{\alpha}$ where $\vec{\alpha}$ is a unit vector. Let $\xi(t)=\frac{m_{\tau_{1}} q_{\tau_{1}}(t)+m_{\tau_{2}} q_{\tau_{2}}(t)}{m_{\tau_{1}}+m_{\tau_{2}}}$ be the center of mass of the $\tau_{1}$-th and $\tau_{2}$-th bodies.

$$
\begin{gathered}
q_{\tau_{1} S_{2}}(t)=\xi(t)+\frac{m_{\tau_{2}}}{m_{\tau_{1}}+m_{\tau_{2}}} r(t), q_{\tau_{2} S_{2}}(t)=\xi(t)-\frac{m_{\tau_{1}}}{m_{\tau_{1}}+m_{\tau_{2}}} r(t) ; \\
q_{j S_{2}}(t)=q_{j}(t), j \notin \mathcal{C} .
\end{gathered}
$$

We consider the deformation of $r(t)$ as

$$
r_{\delta}(t)=r(t)+\delta \phi(t) \vec{s},
$$


where $\vec{s}$ is an appropriate unit vector, $\delta=\frac{\epsilon}{N}$ with $N \geq 2 \max \left\{K_{i n} / U_{i n}, 4\right\}$, and

$$
\phi(t)= \begin{cases}1, & 0 \leq t \leq \delta \\ \frac{\delta+\tilde{N} \delta-t}{\tilde{N} \delta}, & \delta<t \leq \delta+\tilde{N} \delta \\ 0, & \delta+\tilde{N} \delta<t \leq \epsilon\end{cases}
$$

where $K_{\text {in }} / U_{\text {in }}<\tilde{N}<N-1$. The positive $K_{\text {in }}$ and $U_{\text {in }}$ are given in the equations (14) and (15) respectively, which are independent of $\epsilon$.

The collision-free motion $S_{3}$ is denoted by

$$
\begin{gathered}
q_{\tau_{1} S_{3}}(t)=\xi(t)+\frac{m_{\tau_{2}}}{m_{\tau_{1}}+m_{\tau_{2}}} r_{\delta}(t), q_{\tau_{2} S_{3}}(t)=\xi(t)-\frac{m_{\tau_{1}}}{m_{\tau_{1}}+m_{\tau_{2}}} r_{\delta}(t) ; \\
q_{j S_{3}}(t)=q_{j}(t), j \notin \mathcal{C} .
\end{gathered}
$$

We choose $\vec{s}$ to be the unit vector of $(0, \pm 1)$ when $\left\{\tau_{1}, \tau_{2}\right\}=\{2,4\}$ and we choose $\vec{s}$ to be the unit vector of $( \pm 1,0)$ when $\left\{\tau_{1}, \tau_{2}\right\}=\{1,3\}$. The sign will be determined later. So the initial condition of $S_{3}$ satisfies the SPBC.

Now consider the expression of the actions for each path in the time interval $[0, \epsilon]$. They will be decomposed into two parts: the first part $A_{i n}$ is to compute the action of the relative motion of the colliding bodies $m_{\tau_{1}}$ and $m_{\tau_{2}}$; the second part $A_{\text {out }}$ is to compute the action of the remainder, i.e., $A_{j}=A_{j i n}+A_{\text {jout }}$ for $j=1,2,3$. It is easy to know that $A_{1 i n} \geq A_{2 i n}$ since the homothetic collision-ejection orbit is a minimizer. We only need to prove $A_{2 i n}-A_{3 i n}>$ $A_{3 o u t}-A_{1 \text { out }}$ in order to prove $A_{1}>A_{3}$ in $[0, \epsilon]$. We first note that

$$
\begin{gathered}
m_{\tau_{1}}\left|\dot{q}_{\tau_{1} S_{2}}\right|^{2}+m_{\tau_{2}}\left|\dot{q}_{\tau_{2} S_{2}}\right|^{2}=m_{\tau_{1}}\left\langle\dot{\xi}+\frac{m_{\tau_{2}}}{m_{\tau_{1}}+m_{\tau_{2}}} \dot{r}, \dot{\xi}+\frac{m_{\tau_{2}}}{m_{\tau_{1}}+m_{\tau_{2}}} \dot{r}\right\rangle+ \\
m_{\tau_{2}}\left\langle\dot{\xi}-\frac{m_{\tau_{1}}}{m_{\tau_{1}}+m_{\tau_{2}}} \dot{r}, \dot{\xi}-\frac{m_{\tau_{1}}}{m_{\tau_{1}}+m_{\tau_{2}}} \dot{r}\right\rangle=\left(m_{\tau_{1}}+m_{\tau_{2}}\right)|\dot{\xi}|^{2}+\frac{m_{\tau_{1}} m_{\tau_{2}}}{m_{\tau_{1}}+m_{\tau_{2}}}|\dot{r}|^{2} .
\end{gathered}
$$

Then

$$
\begin{gathered}
A_{2 \text { in }}-A_{3 \text { in }}=\int_{0}^{\epsilon} \frac{m_{\tau_{1}} m_{\tau_{2}}}{2\left(m_{\tau_{1}}+m_{\tau_{2}}\right)}\left(|\dot{r}|^{2}-\left|\dot{r}_{\delta}\right|^{2}\right)+m_{\tau_{1}} m_{\tau_{2}}\left(\frac{1}{|r|}-\frac{1}{\left|r_{\delta}\right|}\right) d t \\
A_{3 \text { out }}-A_{1 \text { out }}=\int_{0}^{\epsilon} \sum_{i \notin \mathcal{C} ; \tau_{j} \in \mathcal{C}}\left(\frac{m_{i} m_{\tau_{j}}}{\left|q_{i}-q_{\tau_{j} S_{3}}\right|}-\frac{m_{i} m_{\tau_{j}}}{\left|q_{i}-q_{\tau_{j}}\right|}\right) d t .
\end{gathered}
$$

Now we estimate the bounds for $A_{\text {out }}$. Let $K$ be a large constant satisfying $\mathcal{A}(q) \leq K$. Consider the motion of the mass $m_{j}$ between the arbitrary successive instants $t_{1}$ and $t_{2}$. Because the minimum of the integral $\int_{t_{1}}^{t_{2}} \frac{m_{j}\left|\dot{q}_{j}\right|^{2}}{2} d t$ between given positions $q_{j}\left(t_{1}\right)$ and $q_{j}\left(t_{2}\right)$ is obtained for a constant velocity vector, we can always write $\frac{m_{j}\left|q_{j}\left(t_{2}\right)-q_{j}\left(t_{1}\right)\right|^{2}}{2\left(t_{2}-t_{1}\right)} \leq \int_{t_{1}}^{t_{2}} \frac{m_{j}\left|\dot{q}_{j}\right|^{2}}{2} d t \leq \mathcal{A}(q) \leq K<\infty$. So if $0 \leq t_{1} \leq t_{2} \leq T,\left|q_{j}\left(t_{2}\right)-q_{j}\left(t_{1}\right)\right| \leq\left(\frac{2 K\left(t_{2}-t_{1}\right)}{m_{j}}\right)^{1 / 2}$. Pick up $\epsilon>0$ small such that the two bodies $m_{\tau_{1}}$ and $m_{\tau_{2}}$ will remain at less than twice that distance from the collision point $q_{\tau_{1}}(0)$ all along the time interval $[0, \epsilon]$, i.e. $\left|q_{\tau_{1}}-q_{\tau_{2}}\right| \leq J \sqrt{\epsilon}$, where $J=2(2 K)^{1 / 2} \cdot m_{j}, j \notin \mathcal{C}$ will remain outside of the circle centered at the collision point with radius $D$ and $J \sqrt{\epsilon} \leq J \sqrt{\epsilon_{0}} \ll D$ for a fixed $\epsilon_{0}$. So during the time interval $[0, \epsilon]$, the bodies $m_{j}, j \notin \mathcal{C}$ are outside of the circle with radius $D$ and center $q_{\tau_{1}}(0)$, while the bodies $m_{\tau_{1}}$ and $m_{\tau_{2}}$ are inside the much smaller circle of the same center and radius $J \sqrt{\epsilon}$.

$$
\left|A_{3 \text { out }}-A_{\text {1out }}\right| \leq \int_{0}^{\epsilon} \sum_{i \notin \mathcal{C} ; \tau_{j} \in \mathcal{C}} m_{i} m_{\tau_{j}}\left|\left(\frac{\left|q_{i}-q_{\tau_{j} S_{3}}\right|-\left|q_{i}-q_{\tau_{j}}\right|}{\left|q_{i}-q_{\tau_{j}}\right|\left|q_{i}-q_{\tau_{j} S_{3}}\right|}\right)\right| d t .
$$




$$
\begin{gathered}
\leq \int_{0}^{\epsilon} \sum_{i \notin \mathcal{C} ; \tau_{j} \in \mathcal{C}} m_{i} m_{\tau_{j}}\left(\frac{\left|q_{\tau_{j} S_{3}}-q_{\tau_{j}}\right|}{\left|q_{i}-q_{\tau_{j}}\right|\left|q_{i}-q_{\tau_{j} S_{3}}\right|}\right) d t . \\
\leq \int_{0}^{\epsilon} \sum_{i \notin \mathcal{C} ; \tau_{j} \in \mathcal{C}} m_{i} m_{\tau_{j}}\left(\frac{J \sqrt{\epsilon}}{\left(D-J \sqrt{\epsilon_{0}}\right)^{2}}\right) d t=\frac{4 J}{\left(D-J \sqrt{\epsilon_{0}}\right)^{2}} \epsilon^{\frac{3}{2}}=U_{\text {out }} \epsilon^{\frac{3}{2}} .
\end{gathered}
$$

Let us compute $A_{2 i n}-A_{3 i n}$. By choosing appropriate direction of $\vec{s}$ such that $\langle r, \vec{s}\rangle \geq 0$,

$$
\begin{aligned}
& \int_{0}^{\epsilon} \frac{m_{\tau_{1}} m_{\tau_{2}}}{2\left(m_{\tau_{1}}+m_{\tau_{2}}\right)}\left(|\dot{r}|^{2}-\left|\dot{r}_{\delta}\right|^{2}\right) d t=-\int_{0}^{\epsilon} \frac{m_{\tau_{1}} m_{\tau_{2}}}{2\left(m_{\tau_{1}}+m_{\tau_{2}}\right)}\left(2 \delta \dot{\phi}\langle r, \vec{s}\rangle+(\delta \dot{\phi})^{2}\right) d t \\
& \geq-\int_{\delta}^{\delta+\tilde{N} \delta} \frac{m_{\tau_{1}} m_{\tau_{2}}}{2\left(m_{\tau_{1}}+m_{\tau_{2}}\right)}(\delta \dot{\phi})^{2} d t=-\int_{\delta}^{\delta+\tilde{N} \delta} \frac{m_{\tau_{1}} m_{\tau_{2}}}{2\left(m_{\tau_{1}}+m_{\tau_{2}}\right)}\left(-\frac{1}{\tilde{N}}\right)^{2} d t \\
& \geq-\frac{m_{\tau_{1}} m_{\tau_{2}}}{2\left(m_{\tau_{1}}+m_{\tau_{2}}\right)} \frac{\delta}{\tilde{N}}=-K_{i n} \frac{\delta}{\tilde{N}} \\
& \int_{0}^{\epsilon}\left(\frac{1}{|r|}-\frac{1}{\left|r_{\delta}\right|}\right) d t=\int_{0}^{\epsilon}\left(\frac{1}{|r|}-\frac{1}{\left(|r|^{2}+2 \delta \phi\langle r, \vec{s}\rangle+(\delta \phi)^{2}\right)^{1 / 2}}\right) d t \\
& =\int_{0}^{\epsilon}\left(\frac{2 \delta \phi\langle r, \vec{s}\rangle+(\delta \phi)^{2}}{|r|\left(|r|^{2}+2 \delta \phi\langle r, \vec{s}\rangle+(\delta \phi)^{2}\right)^{1 / 2}\left(|r|+\left(|r|^{2}+2 \delta \phi\langle r, \vec{s}\rangle+(\delta \phi)^{2}\right)^{1 / 2}\right)}\right) d t \\
& \geq \int_{0}^{\delta}\left(\frac{(\delta)^{2}}{|r|(|r|+\delta)(2|r|+\delta)}\right) d t \geq \int_{0}^{\delta}\left(\frac{1}{\gamma\left(\gamma+\delta^{1 / 3}\right)\left(2 \gamma+\delta^{1 / 3}\right)}\right) d t \\
& \geq\left(\frac{1}{\gamma(\gamma+1)(2 \gamma+1)}\right) \delta=U_{i n} \delta
\end{aligned}
$$

where we use the fact $|r| \leq \gamma \delta^{2 / 3}$ in $[0, \delta]$ and $\delta<1$.

So $A_{2 i n}-A_{3 i n}>\left(-K_{\text {in }} \frac{\delta}{N}+U_{\text {in }} \delta\right)=\left(-\frac{K_{i n}}{\tilde{N}}+U_{\text {in }}\right) \frac{\epsilon}{N}>U_{\text {out }} \epsilon^{\frac{3}{2}} \geq A_{3 \text { out }}-A_{1 \text { out }}$ for small $\epsilon$, which implies $A_{1}>A_{3}$.

The action of $S_{3}$ is smaller than the action of $S_{1}$ which contradicts the fact that $S_{1}$ is a minimizer. The contradiction completes the proof that the vector $\vec{a}_{0}$ with binary collision is not a minimizer of $\mathcal{A}$ on $\Gamma$.

\section{CASE TWO: Collision with three or more bodies}

Suppose that $q$ is a local minimizer of $\mathcal{A}$ satisfying the SPBC for $\vec{a}_{0}$. Let the collision subset $\mathcal{C}=\left\{\tau_{1}, \tau_{2}, \cdots, \tau_{k}\right\} \subseteq\{1,2,3,4\}, k=3$ or $k=4$. When $k=3$, it is corresponding to the triple collisions (5) and (6) at $t=0$. When $k=4$, it is corresponding to the total collision (4) at $t=0$. The bodies $m_{\tau_{1}}, m_{\tau_{2}}, \cdots, m_{\tau_{k}}$ start at the collision point $q_{\tau_{1}}(0)=q_{\tau_{2}}(0)=\cdots=$ $q_{\tau_{k}}(0)$ while the other bodies are away. By [31, 33], the configuration of the colliding bodies $m_{\tau_{1}}, m_{\tau_{2}}, \cdots, m_{\tau_{k}}$ is approaching the set of central configurations. We will also build the two following pathes $S_{2}$ (Kepler ejection orbits at the starting point) and $S_{3}$ (the local deformation of $S_{2}$ ) such that $A_{1}>A_{3}$ for sufficiently small time $\epsilon$. Here we consider for triple collision only. By the structural of SPBC, $\{1,3\}$ must be in the collision set $\mathcal{C}$. Without loss of generality, we assume $\left\{\tau_{1}, \tau_{2}\right\}=\{1,3\}$. First, consider the ejection orbits in the starting time interval $[0, \epsilon]$ in 
$S_{2}$. Let $\bar{\omega}=\left(\bar{\omega}_{\tau_{1}}, \bar{\omega}_{\tau_{2}}, \bar{\omega}_{\tau_{3}}\right)$ be the translation of the central configuration $\left(q_{\tau_{1}}(\epsilon), q_{\tau_{2}}(\epsilon), q_{\tau_{3}}(\epsilon)\right)$ by shifting center of mass at origin. By Sundman and Sperling's estimates near collisions $[33,34]$, the homothetic collision-ejection orbit is given by $\omega_{\tau_{i}}(t)=\bar{\omega}_{\tau_{i}} t^{2 / 3}, i=1,2,3$ and $t \in[0, \epsilon]$. Let $\xi(t)=\frac{m_{\tau_{1}} q_{\tau_{1}}(t)+m_{\tau_{2}} q_{\tau_{2}}(t)+m_{\tau_{3}} q_{\tau_{3}}(t)}{m_{\tau_{1}}+m_{\tau_{2}}+m_{\tau_{3}}}$ be the center of mass of the colliding bodies.

$$
q_{\tau_{i} S_{2}}(t)=\xi(t)+\omega_{\tau_{i}}(t), i=1,2,3 ; q_{j S_{2}}(t)=q_{j}(t), j \notin \mathcal{C} .
$$

Now we consider the deformation of $\omega_{\tau_{i}}(t)$ as

$$
\begin{gathered}
\omega_{\tau_{1} \delta}(t)=\omega_{\tau_{1}}(t)+\frac{1}{2 m_{\tau_{1}}} \delta \phi(t) \vec{s}_{1}+\frac{1}{2 m_{\tau_{1}}} \delta \phi(t) \vec{s}_{2}, \\
\omega_{\tau_{2} \delta}(t)=\omega_{\tau_{1}}(t)+\frac{1}{2 m_{\tau_{2}}} \delta \phi(t) \vec{s}_{1}-\frac{1}{2 m_{\tau_{2}}} \delta \phi(t) \vec{s}_{2}, \\
\omega_{\tau_{3} \delta}(t)=\omega_{\tau_{3}}(t)-\frac{1}{m_{\tau_{3}}} \delta \phi(t) \vec{s}_{1},
\end{gathered}
$$

where $\vec{s}_{1}$ and $\vec{s}_{2}$ are appropriate unit vectors, $\delta=\frac{\epsilon}{N}$ with $N \geq 2 \max \left\{K_{i n} / U_{i n}, 4\right\}$, and

$$
\phi(t)= \begin{cases}1, & 0 \leq t \leq \delta \\ \frac{\delta+\tilde{N} \delta-t}{\tilde{N} \delta}, & \delta<t \leq \delta+\tilde{N} \delta \\ 0, & \delta+\tilde{N} \delta<t \leq \epsilon\end{cases}
$$

where $K_{i n} / U_{i n}<\tilde{N}<N-1$. The positive $K_{\text {in }}$ and $U_{\text {in }}$ are given in the equations (18) and (17) respectively and they are independent of $\epsilon$ and $t$. The deformation $S_{3}$ is denoted by

$$
q_{\tau_{i} S_{3}}(t)=\xi(t)+\omega_{\tau_{i} \delta}(t), i=1,2,3 ; q_{j S_{3}}(t)=q_{j S_{2}}(t), j \notin \mathcal{C} .
$$

We choose $\vec{s}_{1}$ to be the unit vector of $(0, \pm 1)$ and $\vec{s}_{2}$ to be the unit vector of $( \pm 1,0)$. The signs of the unite vectors will be determined later. So the initial condition of $S_{3}$ satisfies the SPBC. Now consider the expression of the actions for each path in the time interval $[0, \epsilon]$. They will be decomposed into two parts: the first part $A_{i n}$ is to compute the action of the relative motion of the colliding bodies $m_{\tau_{i}}, \tau_{i} \in \mathcal{C}$; the second part $A_{\text {out }}$ is to compute the action of the remainder. It is easy to know that $A_{1 i n} \geq A_{2 i n}$ since the homothetic collision-ejection orbit is a minimizer. We only need to prove $A_{2 i n}-A_{3 i n}>A_{3 o u t}-A_{1 o u t}$ in order to prove $A_{1}>A_{3}$ in $[0, \epsilon]$.

First of all, we estimate $A_{3 o u t}-A_{1 o u t}$. Consider the motion of the mass $m_{j}$ between the arbitrary successive instants $t_{1}$ and $t_{2}$. Because the minimum of the integral $\int_{t_{1}}^{t_{2}} \frac{m_{j}\left|\dot{q}_{j}\right|^{2}}{2} d t$ between given positions $q_{j}\left(t_{1}\right)$ and $q_{j}\left(t_{2}\right)$ is obtained for a constant velocity vector, we can always write $\frac{m_{j}\left|q_{j}\left(t_{2}\right)-q_{j}\left(t_{1}\right)\right|^{2}}{2\left(t_{2}-t_{1}\right)} \leq \int_{t_{1}}^{t_{2}} \frac{m_{j}\left|\dot{q}_{j}\right|^{2}}{2} d t \leq \mathcal{A}(q) \leq K<\infty$. So if $0 \leq t_{1} \leq t_{2} \leq T, \mid q_{j}\left(t_{2}\right)-$ $q_{j}\left(t_{1}\right) \mid \leq\left(\frac{2 K\left(t_{2}-t_{1}\right)}{m_{j}}\right)^{1 / 2}$. Pick up $\epsilon>0$ small such that the three bodies $m_{\tau_{1}}, m_{\tau_{2}}, \cdots, m_{\tau_{k}}$ will remain in a circle with radius $J \sqrt{\epsilon}$ from the collision point all along the time interval $[0, \epsilon]$, i.e. where $J=\left(2 K / \min \left\{m_{i}\right\}\right)^{1 / 2}$. Then $\left|q_{i}-q_{j}\right| \leq 2 J \sqrt{\epsilon}$ for $i, j \in \mathcal{C} . m_{j}, j \notin \mathcal{C}$ will remain outside of the circle centered at the collision point $q_{\tau_{1}}(0)$ with radius $D$ and $J \sqrt{\epsilon} \leq J \sqrt{\epsilon_{0}} \ll D$. So during the time interval $[0, \epsilon]$, the body $m_{j}$ is outside of the circle with radius $D$ and center $q_{\tau_{1}}(0)$, while the bodies $m_{\tau_{1}}, m_{\tau_{2}}, \cdots, m_{\tau_{k}}$ are inside the much smaller circle with the same center and radius $J \sqrt{\epsilon}$.

$$
\left|A_{3 \text { out }}-A_{1 \text { out }}\right|=\left|\int_{0}^{\epsilon} \sum_{\tau_{i} \in \mathcal{C}, j \notin \mathcal{C}}\left(\frac{m_{\tau_{i}} m_{j}}{\left|q_{\tau_{i} S_{3}}(t)-q_{j S_{3}}(t)\right|}-\frac{m_{\tau_{i}} m_{j}}{\left|q_{\tau_{i}}(t)-q_{j}(t)\right|}\right) d t\right|
$$




$$
\begin{gathered}
=\left|\int_{0}^{\epsilon} \sum_{\tau_{i} \in \mathcal{C}, j \notin \mathcal{C}} \frac{m_{\tau_{i}} m_{j}\left(\left|q_{\tau_{i}}-q_{j}\right|-\left|q_{\tau_{i} S_{3}}-q_{j S_{3}}\right|\right)}{\left|q_{\tau_{i} S_{3}}-q_{j S_{3}}\right|\left|q_{\tau_{i}}-q_{j}\right|} d t\right| \leq \int_{0}^{\epsilon} \sum_{\tau_{i} \in \mathcal{C}, j \notin \mathcal{C}} \frac{m_{\tau_{i}} m_{j}\left(\left|q_{\tau_{i}}-q_{\tau_{i} S_{3}}\right|\right)}{\left|q_{\tau_{i} S_{3}}-q_{j S_{3}}\right|\left|q_{\tau_{i}}-q_{j}\right|} d t \\
\leq \int_{0}^{\epsilon} \frac{8 J \sqrt{\epsilon}}{(D-J \sqrt{\epsilon})^{2}} d t \leq \frac{8 J \epsilon^{3 / 2}}{\left(D-J \sqrt{\epsilon_{0}}\right)^{2}}=U_{\text {out }} \epsilon^{3 / 2} .
\end{gathered}
$$

Now we compute

$$
A_{2 i n}-A_{3 i n}=\int_{0}^{\epsilon} \sum_{\tau_{i} \in \mathcal{C}} \frac{1}{2} m_{\tau_{i}}\left(\left|\dot{q}_{\tau_{i} S_{2}}\right|^{2}-\left|\dot{q}_{\tau_{i} S_{3}}\right|^{2}\right)+\sum_{\tau_{i}<\tau_{j}, \tau_{i} \in \mathcal{C}, \tau_{j} \in \mathcal{C}} \frac{m_{\tau_{i}} m_{\tau_{j}}}{\left|q_{\tau_{i} S_{2}}-q_{\tau_{j} S_{2}}\right|}-\frac{m_{\tau_{i}} m_{\tau_{j}}}{\left|q_{\tau_{i} S_{3}}-q_{\tau_{j} S_{3}}\right|} d t .
$$

Because $m_{\tau_{1}}=m_{\tau_{2}}$ and $\omega_{\tau_{i}}(t)-\omega_{\tau_{j}}(t)=\left(\bar{\omega}_{\tau_{i}}-\bar{\omega}_{\tau_{j}}\right) t^{2 / 3}$ in $[0, \epsilon]$, we are able to pick up the appropriate direction vector $\vec{s}_{1}$ and $\vec{s}_{2}$ such that the inner product

$$
\left\langle\omega_{\tau_{1}}(t)-\omega_{\tau_{2}}(t),\left(\frac{1}{2 m_{\tau_{1}}}+\frac{1}{2 m_{\tau_{2}}}\right) \delta \phi(t) \vec{s}_{2}\right\rangle \geq 0
$$

and

$$
\left\langle\omega_{\tau_{1}}(t)-\omega_{\tau_{3}}(t),\left(\frac{1}{2 m_{\tau_{1}}}+\frac{1}{m_{\tau_{3}}}\right) \delta \phi(t) \vec{s}_{1}+\left(\frac{1}{2 m_{\tau_{1}}}\right) \delta \phi(t) \vec{s}_{2}\right\rangle \geq 0
$$

Because $\bar{\omega}_{\tau_{1}}, \bar{\omega}_{\tau_{2}}$, and $\bar{\omega}_{\tau_{3}}$ is a central configuration, they are either collinear or equilateral triangle. So

$$
\left\langle\omega_{\tau_{2}}(t)-\omega_{\tau_{3}}(t),\left(\frac{1}{2 m_{\tau_{2}}}+\frac{1}{m_{\tau_{3}}}\right) \delta \phi(t) \vec{s}_{1}-\left(\frac{1}{2 m_{\tau_{2}}}\right) \delta \phi(t) \vec{s}_{2}\right\rangle \geq 0 .
$$

For $t \in[0, \delta]$

$$
\begin{gathered}
\left|\omega_{1 \delta}(t)-\omega_{2 \delta}(t)\right| \leq\left|\bar{\omega}_{1}-\bar{\omega}_{2}\right| t^{2 / 3}+\left(\frac{1}{m_{1}}+\frac{1}{2 m_{2}}\right) \delta \leq\left(\left|\bar{\omega}_{1}-\bar{\omega}_{2}\right|+\left(\frac{1}{m_{1}}+\frac{1}{2 m_{2}}\right) \delta^{1 / 3}\right) \delta^{2 / 3} \\
\left(\left|\omega_{1 \delta}(t)-\omega_{2 \delta}(t)\right|+\left|\omega_{1}(t)-\omega_{2}(t)\right|\right) \leq\left(2\left|\bar{\omega}_{1}-\bar{\omega}_{2}\right|+\left(\frac{1}{m_{1}}+\frac{1}{2 m_{2}}\right) \delta^{1 / 3}\right) \delta^{2 / 3} .
\end{gathered}
$$

In particular for $t \leq \delta$,

$$
\begin{gathered}
\frac{m_{\tau_{1}} m_{\tau_{2}}}{\left|q_{\tau_{1} S_{2}}-q_{\tau_{2} S_{2}}\right|}-\frac{m_{\tau_{1}} m_{\tau_{2}}}{\left|q_{\tau_{1} S_{3}}-q_{\tau_{2} S_{3}}\right|}=\frac{m_{\tau_{1}} m_{\tau_{2}}}{\left|\omega_{\tau_{1}}(t)-\omega_{\tau_{2}}(t)\right|}-\frac{m_{\tau_{1}} m_{\tau_{2}}}{\left|\omega_{\tau_{1} \delta}(t)-\omega_{\tau_{2} \delta}(t)\right|} \\
=m_{\tau_{1}} m_{\tau_{2}} \frac{\left|\omega_{\tau_{1} \delta}(t)-\omega_{\tau_{2} \delta}(t)\right|-\left|\omega_{\tau_{1}}(t)-\omega_{\tau_{2}}(t)\right|}{\left|\omega_{\tau_{1}}(t)-\omega_{\tau_{2}}(t)\right|\left|\omega_{\tau_{1} \delta}(t)-\omega_{\tau_{2} \delta}(t)\right|} \\
=m_{\tau_{1}} m_{\tau_{2}} \frac{\left\langle\omega_{\tau_{1}}(t)-\omega_{\tau_{2}}(t),\left(\frac{1}{2 m_{\tau_{1}}}+\frac{1}{2 m_{\tau_{2}}}\right) \delta \phi(t) \vec{s}_{2}\right\rangle+\left|\left(\frac{1}{2 m_{\tau_{1}}}+\frac{1}{2 m_{\tau_{2}}}\right) \delta \phi(t) \vec{s}_{2}\right|^{2}}{\geq \omega_{\tau_{2}}(t)|| \omega_{\tau_{1} \delta}(t)-\omega_{\tau_{2} \delta}(t) \mid\left(\left|\omega_{\tau_{1} \delta}(t)-\omega_{\tau_{2} \delta}(t)\right|+\left|\omega_{\tau_{1}}(t)-\omega_{\tau_{2}}(t)\right|\right)} \\
\geq \frac{m_{\tau_{1}} m_{\tau_{2}}\left(\frac{1}{2 m_{\tau_{1}}}+\frac{1}{2 m_{\tau_{2}}}\right)^{2} \delta^{2}}{\left|\omega_{\tau_{1}}(t)-\omega_{\tau_{2}}(t)\right|\left|\omega_{\tau_{1} \delta}(t)-\omega_{\tau_{2} \delta}(t)\right|\left(\left|\omega_{\tau_{1} \delta}(t)-\omega_{\tau_{2} \delta}(t)\right|+\left|\omega_{\tau_{1}}(t)-\omega_{\tau_{2}}(t)\right|\right)} \\
\frac{m_{\tau_{1}} m_{\tau_{2}}\left(\frac{1}{2 m_{\tau_{1}}}+\frac{1}{2 m_{\tau_{2}}}\right)^{2}}{\left|\bar{\omega}_{\tau_{1}}-\bar{\omega}_{\tau_{2}}\right|\left(\left|\bar{\omega}_{\tau_{1}}-\bar{\omega}_{\tau_{2}}\right|+\left(\frac{1}{2 m_{\tau_{1}}}+\frac{1}{2 m_{\tau_{2}}}\right) \delta^{1 / 3}\right)\left(2\left|\bar{\omega}_{\tau_{1}}-\bar{\omega}_{\tau_{2}}\right|+\left(\frac{1}{2 m_{\tau_{1}}}+\frac{1}{2 m_{\tau_{2}}}\right) \delta^{1 / 3}\right)}
\end{gathered}
$$




$$
\geq \frac{m_{\tau_{1}} m_{\tau_{2}}\left(\frac{1}{2 m_{\tau_{1}}}+\frac{1}{2 m_{\tau_{2}}}\right)^{2}}{\left|\bar{\omega}_{\tau_{1}}-\bar{\omega}_{\tau_{2}}\right|\left(\left|\bar{\omega}_{\tau_{1}}-\bar{\omega}_{\tau_{2}}\right|+\left(\frac{1}{2 m_{\tau_{1}}}+\frac{1}{2 m_{\tau_{2}}}\right)\right)\left(2\left|\bar{\omega}_{\tau_{1}}-\bar{\omega}_{\tau_{2}}\right|+\left(\frac{1}{2 m_{\tau_{1}}}+\frac{1}{2 m_{\tau_{2}}}\right)\right)} .
$$

By similar computations, we have

$$
\begin{gathered}
\int_{0}^{\epsilon} \sum_{\tau_{i}<\tau_{j}, \tau_{i} \in \mathcal{C}, \tau_{j} \in \mathcal{C}} \frac{m_{\tau_{i}} m_{\tau_{j}}}{\left|q_{\tau_{i} S_{2}}-q_{\tau_{j} S_{2}}\right|}-\frac{m_{\tau_{i}} m_{\tau_{j}}}{\left|q_{\tau_{i} S_{3}}-q_{\tau_{j} S_{3}}\right|} d t \\
\geq \int_{0}^{\epsilon} \sum_{\tau_{i}<\tau_{j}, \tau_{i} \in \mathcal{C}, \tau_{j} \in \mathcal{C}} \frac{m_{\tau_{i}} m_{\tau_{j}}\left|\omega_{\tau_{i} \delta}-\omega_{\tau_{i}}+\omega_{\tau_{j}}-\omega_{\tau_{j} \delta}\right|^{2}}{\left|\omega_{\tau_{i}}(t)-\omega_{\tau_{j}}(t)\right|\left|\omega_{\tau_{i} \delta}(t)-\omega_{\tau_{j} \delta}(t)\right|\left(\left|\omega_{\tau_{i} \delta}(t)-\omega_{\tau_{j} \delta}(t)\right|+\left|\omega_{\tau_{i}}(t)-\omega_{\tau_{j}}(t)\right|\right)} d t \\
\quad \geq \int_{0}^{\epsilon} \frac{m_{\tau_{1}} m_{\tau_{2}}\left|\omega_{\tau_{1} \delta}-\omega_{\tau_{1}}+\omega_{\tau_{2}}-\omega_{\tau_{2} \delta}\right|^{2}}{\left|\omega_{\tau_{1}}(t)-\omega_{\tau_{2}}(t)\right|\left|\omega_{\tau_{1} \delta}(t)-\omega_{\tau_{2} \delta}(t)\right|\left(\left|\omega_{\tau_{1} \delta}(t)-\omega_{\tau_{2} \delta}(t)\right|+\left|\omega_{\tau_{1}}(t)-\omega_{\tau_{2}}(t)\right|\right)} d t \\
\quad \int_{0}^{\delta} \frac{m_{\tau_{1}} m_{\tau_{2}}\left(\frac{1}{2 m_{\tau_{1}}}+\frac{1}{2 m_{\tau_{2}}}\right)^{2}}{\left|\bar{\omega}_{\tau_{1}}-\bar{\omega}_{\tau_{2}}\right|\left(\left|\bar{\omega}_{\tau_{1}}-\bar{\omega}_{\tau_{2}}\right|+\left(\frac{1}{2 m_{\tau_{1}}}+\frac{1}{2 m_{\tau_{2}}}\right)\right)\left(2\left|\bar{\omega}_{\tau_{1}}-\bar{\omega}_{\tau_{2}}\right|+\left(\frac{1}{2 m_{\tau_{1}}}+\frac{1}{2 m_{\tau_{2}}}\right)\right)} d t \\
=\frac{m_{\tau_{1}} m_{\tau_{2}}\left(\frac{1}{2 m_{\tau_{1}}}+\frac{1}{2 m_{\tau_{2}}}\right)^{2}}{\left|\bar{\omega}_{\tau_{1}}-\bar{\omega}_{\tau_{2}}\right|\left(\left|\bar{\omega}_{\tau_{1}}-\bar{\omega}_{\tau_{2}}\right|+\left(\frac{1}{2 m_{\tau_{1}}}+\frac{1}{2 m_{\tau_{2}}}\right)\right)\left(2\left|\bar{\omega}_{\tau_{1}}-\bar{\omega}_{\tau_{2}}\right|+\left(\frac{1}{2 m_{\tau_{1}}}+\frac{1}{2 m_{\tau_{2}}}\right)\right)} \delta
\end{gathered}
$$

Since both the centers of mass of central configurations $\omega(t)$ and $\omega_{\delta}(t)$ are at origin, we have the kinetic energy

$$
\begin{gathered}
\sum_{\tau_{i} \in \mathcal{C}} \frac{1}{2} m_{\tau_{i}}\left|\dot{q}_{\tau_{i} S_{2}}\right|^{2}=\sum_{\tau_{i} \in \mathcal{C}} \frac{1}{2} m_{\tau_{i}}\left(|\dot{\xi}|^{2}+\left|\dot{\omega}_{\tau_{i}}(t)\right|^{2}\right) \text { and } \sum_{\tau_{i} \in \mathcal{C}} \frac{1}{2} m_{\tau_{i}}\left|\dot{q}_{\tau_{i} S_{3}}\right|^{2}=\sum_{\tau_{i} \in \mathcal{C}} \frac{1}{2} m_{\tau_{i}}\left(|\dot{\xi}|^{2}+\left|\dot{\omega}_{\tau_{i} \delta}(t)\right|^{2}\right) . \\
\int_{0}^{\epsilon} \sum_{\tau_{i} \in \mathcal{C}} \frac{1}{2} m_{\tau_{i}}\left(\left|\dot{q}_{\tau_{i} S_{2}}\right|^{2}-\left|\dot{q}_{\tau_{i} S_{3}}\right|^{2}\right) d t=\int_{0}^{\epsilon} \sum_{\tau_{i} \in \mathcal{C}} \frac{1}{2} m_{\tau_{i}}\left(\left|\dot{\omega}_{\tau_{i}}(t)\right|^{2}-\left|\dot{\omega}_{\tau_{i} \delta}(t)\right|^{2}\right) d t \\
\geq-\int_{\delta}^{\delta+\tilde{N} \delta} \frac{1}{2} m_{\tau_{1}}\left|\frac{1}{2 m_{\tau_{1}}} \delta \dot{\phi}(t) \vec{s}_{1}+\frac{1}{2 m_{\tau_{1}}} \delta \dot{\phi}(t) \vec{s}_{2}\right|^{2}+\frac{1}{2} m_{\tau_{2}}\left|\frac{1}{2 m_{\tau_{2}}} \delta \dot{\phi}(t) \vec{s}_{1}-\frac{1}{2 m_{\tau_{2}}} \delta \dot{\phi}(t) \vec{s}_{2}\right|^{2} \\
+\frac{1}{2} m_{\tau_{3}}\left|\frac{1}{m_{\tau_{3}}} \delta \dot{\phi}(t) \vec{s}_{1}\right|^{2} d t \\
\geq-\left(\frac{1}{4 m_{\tau_{1}}}+\frac{1}{4 m_{\tau_{2}}}+\frac{1}{2 m_{\tau_{3}}}\right)\left(\frac{\delta}{\tilde{N}}\right) \equiv-K_{i n} \frac{\delta}{\tilde{N}} .
\end{gathered}
$$

From the above estimations (16),(18) and (17), by picking small enough $\epsilon$, we have

$$
A_{2 i n}-A_{3 i n} \geq\left(U_{\text {in }}-\frac{K_{\text {in }}}{\tilde{N}}\right) \delta=\left(U_{\text {in }}-\frac{K_{\text {in }}}{\tilde{N}}\right) \frac{\epsilon}{N} \geq U_{\text {out }} \epsilon^{3 / 2}>A_{3 o u t}-A_{1 o u t} .
$$

The inequality implies that the action of the deformation is smaller than the action of the minimizer. This completes the proof that the minimizer can not have the triple collisions. 
The proof of theorem 2.5. By theorem 2.3, any path $q^{*}(t)$ corresponding to a local minimizer $\vec{a}_{0}$ is a classic solution in the interval $[0, T]$. We prove this theorem by two steps. First, we prove that it can be extended to a periodic solutions. Second, we prove that there exists a local minimizer $\vec{a}_{0}$ which produce the star pentagon solution.

Because $q^{*}(t)$ is a classic solution of Newton's equation $(2)$ on $(0, T)$, it is easy to check that $q(t)$ is a classical solution in each interval $((n-1) T, n T)$ for any given positive integer $n$. To prove $q(t)$ is a classical solution for all real $t$, we need prove that $q(t)$ is connected very well at $t=n T$ for any integer $n$, i.e. $\lim _{t \rightarrow(n T)^{-}} q(t)=\lim _{t \rightarrow(n T)^{+}} q(t)$ and $\lim _{t \rightarrow(n T)^{-}} \dot{q}(t)=$ $\lim _{t \rightarrow(n T)^{+}} \dot{q}(t)$. By the structure of the extension equation (11), we only need prove it for $n=1$ and $n=2$. By the SPBC, we have $\lim _{t \rightarrow(n T)^{-}} q(t)=\lim _{t \rightarrow(n T)^{+}} q(t)$ at $n=1$ and $n=2$. That $\lim _{t \rightarrow(n T)^{-}} \dot{q}(t)=\lim _{t \rightarrow(n T)^{+}} \dot{q}(t)$ at $n=1$ and $n=2$ is equivalent to the relations given by (19) and (20) below.

$$
\begin{array}{ll}
\dot{q}_{1}(T)=\left(\dot{q}_{21}(T),-\dot{q}_{22}(T)\right) R(2 \theta), & \dot{q}_{2}(T)=\left(\dot{q}_{11}(T),-\dot{q}_{12}(T)\right) R(2 \theta), \\
\dot{q}_{3}(T)=\left(\dot{q}_{41}(T),-\dot{q}_{42}(T)\right) R(2 \theta), & \dot{q}_{4}(T)=\left(\dot{q}_{31}(T),-\dot{q}_{32}(T)\right) R(2 \theta),
\end{array}
$$

and at $t=2 T$,

$$
\dot{q}_{11}(0)=\dot{q}_{31}(0), \dot{q}_{12}(0)=-\dot{q}_{32}(0), \dot{q}_{22}(0)=\dot{q}_{42}(0)=0 .
$$

Since $\vec{a}_{0} \in \Gamma$ is a minimizer of $\tilde{\mathcal{A}}(\vec{a})$ over $\Gamma, q^{*}$ is a minimizer of $\mathcal{A}$ over the function space $\mathcal{P}(\mathbf{A}, \mathbf{B})$ by lemma 2.4. Here we use $q$ for $q^{*}$ by our extension formula (11). Consider an admissible variation $\xi \in \mathcal{P}(\mathbf{A}, \mathbf{B})$ with $\xi(0) \in \mathbf{A}$ and $\xi(T) \in \mathbf{B}$, then the first variation $\delta_{\xi} \mathcal{A}(q)$ is computed as:

$$
\begin{gathered}
\delta_{\xi} \mathcal{A}(q)=\lim _{\delta \rightarrow 0} \frac{\mathcal{A}(q+\delta \xi)-\mathcal{A}(q)}{\delta} \\
=\int_{0}^{T} \frac{1}{2} \sum_{i=1}^{4} \lim _{\delta \rightarrow 0} m_{i} \frac{\left|\dot{q}_{i}+\delta \dot{\xi}_{i}\right|^{2}-\left|\dot{q}_{i}\right|^{2}}{\delta}+\lim _{\delta \rightarrow 0} \frac{U(q+\delta \xi)-U(q)}{\delta} d t \\
=\int_{0}^{T}\left(\sum_{i=1}^{4} m_{i}<\dot{q}_{i}, \dot{\xi}_{i}>+\sum_{i=1}^{4}<\frac{\partial}{\partial q_{i}}(U(q(t))), \xi_{i}>\right) d t \\
=\sum_{i=1}^{4} m_{i}<\dot{q}_{i}, \xi_{i}>\left.\right|_{t=0} ^{t=T}+\int_{0}^{T}<-m_{i} \ddot{q}_{i}+\frac{\partial}{\partial q_{i}}(U(q(t))), \xi_{i}>d t .
\end{gathered}
$$

Because the first variation $\delta_{\xi} \mathcal{A}(q)$ vanishes for any $\xi, q$ satisfies Newton's equation (2) and $m_{i}=1, i=1,2,3,4$, we have

$$
\delta_{\xi} \mathcal{A}(q)=\sum_{i=1}^{4}\left(<\dot{q}_{i}(T), \xi(T)>\right)-\sum_{i=1}^{4}\left(<\dot{q}_{i}(0), \xi(0)>\right)=0
$$

For $i=1,2,3$, let $\xi^{(i)}(t) \in \mathcal{P}(\mathbf{A}, \mathbf{B})$ satisfy $\xi^{(i)}(T)=0$ and $\xi^{(i)}(0)=\left(\begin{array}{cc}a_{1} & a_{2} \\ 0 & -a_{3} \\ -a_{1} & a_{2} \\ 0 & -2 a_{2}+a_{3}\end{array}\right)$,

where $a_{i}=1, a_{j}=0$ if $j \neq i$. Then

$$
\begin{gathered}
\delta_{\xi^{(1)}} \mathcal{A}(q)=-\left(\dot{q}_{11}(0)-\dot{q}_{31}(0)\right)=0, \\
\delta_{\xi^{(2)}} \mathcal{A}(q)=-\left(\dot{q}_{12}(0)+\dot{q}_{32}(0)-2 \dot{q}_{42}(0)\right)=0,
\end{gathered}
$$




$$
\delta_{\xi^{(3)}} \mathcal{A}(q)=-\left(-\dot{q}_{22}(0)+\dot{q}_{42}(0)\right)=0 .
$$

By using $\sum_{i=1}^{4} \dot{q}_{i 1}(0)=0$ and $\sum_{i=1}^{4} \dot{q}_{i 2}(0)=0$, we can prove that relation (20) holds after simple calculation.

For $i=4,5,6$, let $\xi^{(i)}(t) \in \mathcal{P}(\mathbf{A}, \mathbf{B})$ satisfy $\xi^{(i)}(0)=0$ and $\xi^{(i)}(T)=\left(\begin{array}{cc}-a_{5} & a_{4} \\ a_{5} & a_{4} \\ -a_{6} & -a_{4} \\ a_{6} & -a_{4}\end{array}\right) R(\theta)$, where $a_{i}=1, a_{j}=0$ if $j \neq i$. Then

$$
\begin{gathered}
\delta_{\xi^{(4)}} \mathcal{A}(q)=<\dot{q}_{1}(T)+\dot{q}_{2}(T),(0,1) R(\theta)>+<\dot{q}_{3}(T)+\dot{q}_{4}(T),(0,-1) R(\theta)> \\
=\left(\dot{q}_{11}+\dot{q}_{21}\right) \sin (\theta)+\left(\dot{q}_{12}+\dot{q}_{22}\right) \cos (\theta)-\left(\dot{q}_{31}+\dot{q}_{41}\right) \sin (\theta)-\left(\dot{q}_{32}+\dot{q}_{42}\right) \cos (\theta)=0 \\
\delta_{\xi^{(5)}} \mathcal{A}(q)=<\dot{q}_{1}(T),(-1,0) R(\theta)>+<\dot{q}_{2}(T),(1,0) R(\theta)> \\
=-\dot{q}_{11} \cos (\theta)+\dot{q}_{12} \sin \theta+\dot{q}_{21} \cos (\theta)-\dot{q}_{22} \sin (\theta)=0 ; \\
\delta_{\xi^{(6)}} \mathcal{A}(q)=<\dot{q}_{3}(T),(-1,0) R(\theta)>+<\dot{q}_{4}(T),(1,0) R(\theta)> \\
=-\dot{q}_{31} \cos (\theta)+\dot{q}_{32} \sin \theta+\dot{q}_{41} \cos (\theta)-\dot{q}_{42} \sin (\theta)=0,
\end{gathered}
$$

where the derivatives are taken at $t=T$.

Let

$$
\begin{aligned}
& A_{i 1}=\dot{q}_{i 1}-\dot{q}_{(i+1) 1} \cos (2 \theta)+\dot{q}_{(i+1) 2} \sin (2 \theta), \\
& A_{i 2}=\dot{q}_{i 2}+\dot{q}_{(i+1) 1} \sin (2 \theta)+\dot{q}_{(i+1) 2} \cos (2 \theta), \\
& A_{j 1}=\dot{q}_{j 1}-\dot{q}_{(j-1) 1} \cos (2 \theta)+\dot{q}_{(j-1) 2} \sin (2 \theta), \\
& A_{j 2}=\dot{q}_{j 2}+\dot{q}_{(j-1) 1} \sin (2 \theta)+\dot{q}_{(j-1) 2} \cos (2 \theta),
\end{aligned}
$$

for $i=1,3$ and $j=2,4$. Because $\sum_{i=1}^{4} \dot{q}_{i k}=0$ for $k=1,2$, we have

$$
A_{11}+A_{21}+A_{31}+A_{41}=0, \quad A_{12}+A_{22}+A_{32}+A_{42}=0 .
$$

By using the trigonometric identities $\cos (\theta)=\cos (2 \theta) \cos (\theta)+\sin (2 \theta) \sin (\theta)$ and $\sin (\theta)=$ $\sin (2 \theta) \cos (\theta)-\cos (2 \theta) \sin (\theta)$, from equation $(22)$, we have

$\dot{q}_{11} \sin (\theta)+\dot{q}_{21}(\sin (2 \theta) \cos (\theta)-\cos (2 \theta) \sin (\theta))+\dot{q}_{12} \cos (\theta)+\dot{q}_{22}(\cos (2 \theta) \cos (\theta)+\sin (2 \theta) \sin (\theta))-$ $\dot{q}_{31} \sin (\theta)-\dot{q}_{41}(\sin (2 \theta) \cos (\theta)-\cos (2 \theta) \sin (\theta))-\dot{q}_{32} \cos (\theta)-\dot{q}_{42}(\cos (2 \theta) \cos (\theta)+\sin (2 \theta) \sin (\theta))=0$, which is

$$
A_{11} \sin (\theta)+A_{12} \cos \theta-A_{31} \sin (\theta)-A_{32} \cos (\theta)=0,
$$

Similarly from equation (22), we also have

$$
A_{21} \sin (\theta)+A_{22} \cos \theta-A_{41} \sin (\theta)-A_{42} \cos (\theta)=0,
$$

$$
A_{21} \sin (\theta)+A_{22} \cos \theta-A_{31} \sin (\theta)-A_{32} \cos (\theta)=0 .
$$


From equation (23) and (24) we have

$$
\begin{aligned}
& A_{11} \cos (\theta)-A_{12} \sin \theta=0, \\
& A_{21} \cos (\theta)-A_{22} \sin \theta=0, \\
& A_{31} \cos (\theta)-A_{32} \sin \theta=0, \\
& A_{41} \cos (\theta)-A_{42} \sin \theta=0 .
\end{aligned}
$$

The equations (26), (29), (31) imply

$$
A_{11}=A_{31}, A_{12}=A_{32} .
$$

The equations (27), (30), (32) imply

$$
A_{21}=A_{41}, A_{22}=A_{42} .
$$

The equations (28), (29), (30) and (33) imply

$$
A_{11}=A_{21}, A_{12}=A_{22} .
$$

Then the above three equations and equation (25) imply that $A_{k j}=0$ for $k=1,2,3,4$ and $j=1,2$. Because the relations (19) is equivalent to $A_{k j}=0$, we complete the proof that $q(t)$ connects very well at $t=2 T$.

Now we prove that there exists a minimizing path which is different from the circular motion. The circular motion can be obtained by extending the corresponding minimizing path of a particular local minimizer $\vec{a}^{\circ}$ in $\Gamma$. Let $q^{\circ}(t)$ be the corresponding path of $\vec{a}^{\circ}$ on $[0, T]$ which can be extended to a circular solution. It is not hard to get the exact formula for action $\mathcal{A}\left(q^{\circ}(t)\right)$ (see equation (38) in Appendix A):

$$
\mathcal{A}\left(q^{\circ}(t)\right)=3(2)^{-\frac{1}{3}} U_{0}^{\frac{2}{3}} T^{\frac{1}{3}}\left(\theta-\frac{\pi}{4}\right)^{\frac{2}{3}},
$$

where $U_{0}=2 \sqrt{2}+1$ is the constant value of the potential function for the four-body problem with equal masses 1 at the vertex of unit square. For $\theta=\frac{2 \pi}{5}$ and $T=1$,

$$
\mathcal{A}\left(q^{\circ}(t)\right) \approx 3.528734094 \text {. }
$$

We assume that the test path $\bar{q}(t)$ is formed by connecting the straight line with constant velocity from the starting configuration Qstart to the ending configuration Qend for the given $\vec{a}$. We now evaluate the action $\mathcal{A}(\bar{q}(t))$ of the test path $\bar{q}(t)$ for $T=1$ and $\vec{a}=[1.0597,1.7696$, $0.8094,0.7536,1.1032,2.4398]$. It is not hard to compute the action over the time $[0, T]$. Although the corresponding action can be calculated by hand, it is computed by a Matlab program (see Appendix B).

$$
\mathcal{A}(\bar{q}(t))=3.2484<\mathcal{A}\left(q^{\circ}(t)\right) .
$$

So there exists a local minimizer $\vec{a}_{0}$ such that $\tilde{\mathcal{A}}\left(\vec{a}_{0}\right)<\mathcal{A}(\bar{q}(t))<\mathcal{A}\left(q^{\circ}(t)\right)$. Then the corresponding minimizing path $q^{*}$ of $\vec{a}_{0}$ produces the star pentagon solution. From the extension equation (11), it is easy to prove other properties of the main theorem 1.1. Because $\sigma=[2,3,4,1]$ is the permutation with $\sigma^{4}(q(t))=q(t)$ and $\theta=\frac{2 \pi}{5}$ and the least common multiple of 4 and 5 is 20, the minimum period of the solution is $\mathcal{T}=40 \mathrm{~T}$ by extension equation (11). When $k=5, q(t)=\sigma^{5}(q(t-10 T)) R(10 \theta)=\sigma(q(t-10 T))$ for $t \in(10 T, 12 T]$ which implies that the solution is choreographic. 


\section{Linearly Stability of Star Pentagon}

Suppose that $\gamma(t)$ is a $\mathcal{T}$-periodic solution to the Hamiltonian system $\dot{\gamma}=J \nabla H(\gamma)$, where $J=\left[\begin{array}{ll}0 & I \\ -I & 0\end{array}\right]$ is the standard symplectic matrix and $I$ is the appropriately sized identity matrix. Let $X(t)$ be the fundamental matrix solution to

$$
\dot{\xi}=J D^{2} H(\gamma(t)) \xi, \quad \xi(0)=I .
$$

$X(t)$ is symplectic and satisfies $X(t+\mathcal{T})=X(t) X(\mathcal{T})$ for all $t$. The matrix $X(\mathcal{T})$ is called the monodromy matrix whose eigenvalues, the characteristic multipliers, determine the linear stability of the periodic solution. Since every integral in the $n$-body problem yields a multiplier of +1 , there are eight +1 multipliers for a periodic orbit in the planar problem. It is natural to define the linear stability of a periodic solution by examining stabiltiy on the reduced quotient space.

Definition 4.1. A periodic solution of the planar $n$-body problem has eight trivial characteristic multipliers of +1 . The solution is spectrally stable if the remaining multipliers lie on the unit circle and linearly stable if, in addition, the monodromy matrix $X(T)$ restricted to the reduced space is diagonalizable.

There are certain several ways to study the linear stability of periodic solutions, especially the ways developed in study the stability of the figure eight solution by Roberts [30] (a dimension reduction) and by Kapela-Simó [21] (a computer-assisted treatment by interval arithmetic). We will apply the standard symplectic reduction by removing the first integrals to reduce the dimension of the monodromy matrix. This is a similar approach as in [30]. However the monodromy matrix for planar 4-body problem still has 8 dimension even if we can separate all those characteristic multipliers of +1 . Our method can be applied to any periodic solution of planar 4-body problem.

\subsection{Classical Symplectic Reduction.}

Here we apply standard symplectic transform to reduce Hamiltonian system to a 10 dimension Hamiltonian system. The monodromy matrix of the periodic solution $\gamma(t)$ in the reduced system has a pair of +1 eigenvalues and the remaining eight eigvalues must be on the unit circle if the solution is linearly stable.

To eliminate the trivial +1 multipliers of a periodic solution, we use Jacobian coordinates and symplectic polar coordinates (see chapter 7 in [29]). Denote $p_{i}=m_{i} \dot{q}_{i}$ as the momentum coordinates and let $\mu_{i}=\sum_{j=1}^{i} m_{j}$ and $M_{i}=\frac{m_{i} \mu_{i-1}}{\mu_{i}}$. Then let

$$
\begin{array}{ll}
g_{4}=\frac{m_{4} q_{4}+m_{3} q_{3}+m_{2} q_{2}+m_{1} q_{1}}{m_{1}+m_{2}+m_{3}+m_{4}}, & G_{4}=p_{4}+p_{3}+p_{2}+p_{1} ; \\
u_{2}=q_{2}-q_{1}, & v_{2}=\frac{\mu_{1} p_{2}}{\mu_{2}}-\frac{m_{2} p_{1}}{\mu_{2}} ; \\
u_{3}=q_{3}-\frac{m_{2} q_{2}+m_{1} q_{1}}{m_{1}+m_{2}}, & v_{3}=\frac{\mu_{2} p_{3}}{\mu_{3}}-\frac{m_{3}\left(p_{2}+p_{1}\right)}{\mu_{3}} \\
u_{4}=q_{4}-\frac{m_{3} q_{3}+m_{2} q_{2}+m_{1} q_{1}}{m_{1}+m_{2}+m_{3}}, & v_{4}=\frac{\mu_{3} p_{4}}{\mu_{4}}-\frac{m_{4}\left(p_{3}+p_{2}+p_{1}\right)}{\mu_{4}} .
\end{array}
$$

The new Hamiltonian is

$$
H_{2}\left(u_{2}, u_{3}, u_{4}, v_{2}, v_{3}, v_{4}\right)=\frac{v_{2}^{2}}{2 M_{2}}+\frac{v_{3}^{2}}{2 M_{3}}+\frac{v_{4}^{2}}{2 M_{4}}-U_{2} .
$$


$U_{2}$ is the corresponding potential energy in the new coordinates and similarly $U_{3}, U_{4}$ in the below are the potential energy in the different cooordinates. The new Hamiltonian is independent of $g_{4}$ and $G_{4}$, the center of mass and total linear momentum respectively. This reduces the dimension by four from 16 to 12 .

Next we change to symplectic polar coordinates to eliminate the integrals due to the angular momentum and rotational symmetry. Set

$$
\begin{gathered}
u_{i}=\left(r_{i} \cos \left(\theta_{i}\right), r_{i} \sin \left(\theta_{i}\right)\right) \\
v_{i}=\left(R_{i} \cos \left(\theta_{i}\right)-\frac{\Theta_{i}}{r_{i}} \sin \left(\theta_{i}\right), R_{i} \sin \left(\theta_{i}\right)+\frac{\Theta_{i}}{r_{i}} \cos \left(\theta_{i}\right)\right)
\end{gathered}
$$

for $i=2,3,4$. Then the new Hamiltonian becomes

$$
H_{3}=\frac{R_{2}^{2} r_{2}^{2}+\Theta_{2}^{2}}{2 M_{2} r_{2}^{2}}+\frac{R_{3}^{2} r_{3}^{2}+\Theta_{3}^{2}}{2 M_{3} r_{3}^{2}}+\frac{R_{4}^{2} r_{4}^{2}+\Theta_{4}^{2}}{2 M_{4} r_{4}^{2}}-U_{3} .
$$

Note that the Hamiltonian $H_{3}$ has only terms of difference angles. This suggests making a final symplectic change of coordinates by leaving the radial variables alone. Use the generating function $S=\Theta_{2} x_{2}+\Theta_{3}\left(x_{3}+x_{2}\right)+\Theta_{4}\left(x_{4}+x_{3}+x_{2}\right)$, and so

$$
\begin{aligned}
& \theta_{2}=x_{2}, \theta_{3}=x_{3}+x_{2}, \theta_{4}=x_{4}+x_{3}+x_{2} ; \\
& \Theta_{2}=X_{2}-X_{3}, \Theta_{3}=X_{3}-X_{4} ; \Theta_{4}=X_{4} .
\end{aligned}
$$

The new Hamiltonian will be independent of $x_{2}$ which means that $X_{2}=\Theta_{2}+\Theta_{3}+\Theta_{4}$ (total angular momentum) is an integral, and $x_{2}$ is an ignorable variable. Setting $X_{2}=c$ and plugging into the Hamiltonian $H_{3}$ yields

$$
H_{4}=\frac{R_{2}^{2} r_{2}^{2}+\left(c-X_{3}\right)^{2}}{2 M_{2} r_{2}^{2}}+\frac{R_{3}^{2} r_{3}^{2}+\left(X_{3}-X_{4}\right)^{2}}{2 M_{3} r_{3}^{2}}+\frac{R_{4}^{2} r_{4}^{2}+X_{4}^{2}}{2 M_{4} r_{4}^{2}}-U_{4} .
$$

This reduces the system to 10 dimensions, with the variables $z=\left(r_{2}, r_{3}, r_{4}, x_{3}, x_{4}, R_{2}, R_{3}, R_{4}\right.$, $\left.X_{3}, X_{4}\right)$.

Because $H_{4}$ is a Hamiltonian system, the monodromy matrix $X(\mathcal{T})$ is symplectic. Its periodic solution $\gamma(t)$ will generate an eigenvector of $X(\mathcal{T})$. In fact, $\gamma(t)$ is a solution of $\dot{z}=J \nabla H_{4}(z)$ with initial condition $z(0)=\gamma(0)$. Then $\ddot{\gamma}(t)=J D^{2} H_{4}(\gamma(t)) \dot{\gamma}(t)$. This implies that $\dot{\gamma}(t)$ satisfies the associated linear system

$$
\dot{\xi}=J D^{2} H_{4}(\gamma(t)) \xi, \quad \xi(0)=\dot{\gamma}(0) .
$$

Since $X(t)$ is the fundamental solution of the above linear system, $\dot{\gamma}(t)=X(t) \dot{\gamma}(0)$, which implies $X(\mathcal{T}) \dot{\gamma}(0)=\dot{\gamma}(\mathcal{T})=\dot{\gamma}(0)$. Because $X(\mathcal{T})$ is symplectic, the eigenvalue +1 must have even multiplicity. So the Monodromy matrix has at least two +1 multipliers, leaving the remaining eight eigenvalues to determine the linear stability of the periodic solution. Because the eigenvalues of a symplectic matrix occur in quadruples $\left(\lambda, \lambda^{-1}, \bar{\lambda}, \bar{\lambda}^{-1}\right)$, we have the following lemma.

Lemma 4.2. Let $X$ be a symplectic matrix and $W=\frac{1}{2}\left(X+X^{-1}\right)$. Then the eigenvalues of $X$ are all on the unit circle if and only if all of the eigenvalues of $W$ are real and in $[-1,1]$.

Proof. The lemma and its proof are similar to Lemma 4.1 in Roberts' paper [30]. We prove it here for the sake of completeness. Suppose that $\vec{v}$ is an eigenvector of the symplectic matrix 
$X$ with eigenvalue $\lambda$, i.e. $X \vec{v}=\lambda \vec{v}$. Then $X^{-1} \vec{v}=\lambda^{-1} \vec{v} . W \vec{v}=\frac{1}{2}\left(X+X^{-1}\right) \vec{v}=\frac{1}{2}\left(\lambda+\lambda^{-1}\right) \vec{v}$ from which it follows that $\frac{1}{2}\left(\lambda+\lambda^{-1}\right)$ is an eigenvalue of $W$. The map $f: \mathcal{C} \mapsto \mathcal{C}$ given by $f(\lambda)=\frac{1}{2}\left(\lambda+\lambda^{-1}\right)$ takes the unit circle onto the real interval $[-1,1]$ while mapping the exterior of the unit disk homeomorphically onto $\mathcal{C} \backslash[-1,1]$. The lemma follows this assertion immediately.

Because the eigenvalue pairs $\lambda$ and $\lambda^{-1}$ of $X$ is mapped to the same eigenvalue $\frac{1}{2}\left(\lambda+\lambda^{-1}\right)$ of $W$, the multiplicity of eigenvalues of $W$ must be at least two. The two +1 multipliers is still mapped to +1 with multiplicity two. The remaining eight non-one eigenvalues on the unit circle of $X$ for linear stable periodic solution have been mapped to four pairs of real eigenvalues in $(-1,1)$.

\subsection{Numerical Calculations}

Numerically, a MATLAB program was written using a Runge-Kutta-Fehlberg method with local truncation error of order four to compute the monodromy matrix $X(\mathcal{T})$ of the reduced linearized Hamiltonian $\mathrm{H}_{4}$ for a periodic solution of planar 4-body problem. Then we compute $W=\frac{1}{2}\left(X+X^{-1}\right)$ and its eigenvalues. In order to conclude the stability, we first need to improve the estimates of our SPBC $\vec{a}_{0}$ and initial conditions of a periodic solutions. As we described in section 2, there are two steps in searching a solution satisfying SPBC.The first step is to find a solution for a fixed boundary and the second step is to vary the boundary to find a minimizer. More details on the algorithm is given in appendix C. In this way, we can easily get a good approximation of the initial conditions of the Star Pentagon and other solutions with an absolute error tolerance of $10^{-12}$.

In particular, the initial conditions for the star pentagon choreographic solution are given in the figure 1. We compute $W$ using step size 2.6e-5 and its four pairs of eigenvalues other than the multipliers +1 are $[0.761479,0.235862,-0.299445,-0.456737]$. The four pairs of eigenvalues are real and distinct in $(-1,1)$. Returning to the full monodromy matrix, the corresponding eigenvalues are distinct and on the unit circle. Therefore, the star pentagon choreographic solution is linearly stable.

In order to check whether the global error of the numerical eigenvalues is under control, we compute $W$ and its eigenvalues with different step sizes. For example using step size 5.2e-5, the four pairs of eigenvalues are $[0.761189,0.235968,-0.299247,-0.456741]$. The maximum of absolute value of the difference between the eigenvalues by step size 5.2e-5 and the eigenvalues by step size $2.6 e-5$ are 0.00029 which verify that these results are within the expected accuracy. Table 1 shows the four pairs of eigenvalues ordered $\lambda_{1} \geq \lambda_{2} \geq \lambda_{3} \geq \lambda_{4}$. Because we need to linearize the system along periodic solutions, the accuracy of the eigenvalues of monodromy matrix strongly depends on the accuracy of periodic solutions. We choose small step size to make the accuracy of the periodic solutions higher. Here we list the step size and its computation time when we compute $W$ and its eigenvalues. We also compare the eigenvalues with the eigenvalues by step size $2.6 e-5$ and the difference are all within the expected accuracy.

Remark 4.3. Without the symplectic reduction, the original Hamiltonian system of the planar four-body problem has 16 dimension. To check the stability of a periodic solution, one can directly compute the eigenvalues of its monodromy matrix. Thanks a Matlab Program by Professor Robert Vanderbei, the largest absolute values of all 16 eigenvalues for the star pentagon solution and other stable solutions in this paper are all 1.0000 which lead to the same stability 


\begin{tabular}{|c|c|c|c|c|c|c|}
\hline Step size $\Delta t$ & Comp. Time & $\lambda_{1}$ & $\lambda_{2}$ & $\lambda_{3}$ & $\lambda_{4}$ & $\max _{i}\left|\lambda_{i}-\lambda_{i}^{0}\right|$ \\
\hline $2.8 \mathrm{e}-3$ & $4.3 \mathrm{~s}$ & 0.729545 & 0.247271 & -0.282346 & -0.457173 & 0.031934 \\
$6.1 \mathrm{e}-4$ & $19.4 \mathrm{~s}$ & 0.754903 & 0.238246 & -0.295721 & -0.456820 & 0.006576 \\
$3.1 \mathrm{e}-4$ & $39.8 \mathrm{~s}$ & 0.758309 & 0.237014 & -0.297621 & -0.456777 & 0.00317 \\
$1.5 \mathrm{e}-4$ & $78.1 \mathrm{~s}$ & 0.760032 & 0.236388 & -0.298592 & -0.456755 & 0.001447 \\
$7.8 \mathrm{e}-5$ & $155.2 \mathrm{~s}$ & 0.760899 & 0.236073 & -0.299083 & -0.456744 & 0.00058 \\
$5.2 \mathrm{e}-5$ & $234.8 \mathrm{~s}$ & 0.761189 & 0.235968 & -0.299247 & -0.456741 & 0.00029 \\
$2.6 \mathrm{e}-5$ & $470.9 \mathrm{~s}$ & 0.761479 & 0.235862 & -0.299412 & -0.456737 & 0 \\
\hline
\end{tabular}

Table 1: Stability Analysis for Star Pentagon Choreographic Solution. The last column is the difference of eigenvalues from our estimate by step size $2.6 e-5 . \quad \lambda_{i}^{0}$ is the eigenvalue in the last row.

result.

\section{Classification of solutions and rotation angles $\theta$}

Theorem 1.3 can be restated as following theorem with detail classifications.

Theorem 5.1. There exist $\theta_{0}$ and $\theta_{1}$ satisfying $\frac{\pi}{4}<\theta_{0}<\frac{\pi}{2}<\theta_{1}<\frac{3 \pi}{4}$ such that for any $\theta \in\left(\theta_{0}, \theta_{1}\right)$ and $\theta \neq \frac{\pi}{2}$, there exists at least one local minimizer $\vec{a}_{0} \in \Gamma$ for the variational problem (10) with the SPBC and its corresponding minimizing path $q^{*}(t)$ connecting $q(0)$ and $q(T)$ can be extended to a non-circular classical Newtonian solution $q(t)$ by the same extension as (11) in theorem 2.5. Each curve $q_{i}(t), t \in[8 k T,(8 k+8) T]$ is called a side of the orbit since the orbit of the solution is assembled out the sides by rotation only. The non-circular solution $q(t)$ can be classified as follows.

(1) [Quasi-Periodic Solutions] $q(t)$ is a quasi-periodic solution if $\theta$ is not commensurable with $\pi$.

(2) [Periodic Solutions] $q(t)$ is a periodic solution if $\theta=\frac{P}{Q} \pi$, where the positive integers $P$ and $Q$ are relatively prime.

- When $Q \equiv 0 \bmod 4$, the periodic solution $q(t)$ is a non-choreographic solution. Each closed curve has $\frac{Q}{4}$ sides. The minimum period is $\mathcal{T}=2 Q T$.

- When $Q \equiv 1 \bmod 4$, the periodic solution $q(t)$ is a choreographic solution. The closed curve has $Q$ sides. The minimum period is $\mathcal{T}=8 Q T$. The four bodies chase each other on the closed curve in the order of $q_{1}, q_{2}, q_{3}, q_{4}$, and then $q_{1}$, i.e. $q_{1}(t+2 Q T)=q_{2}(t)$, $q_{2}(t+2 Q T)=q_{3}(t), q_{3}(t+2 Q T)=q_{4}(t)$, and $q_{4}(t+2 Q T)=q_{1}(t)$.

- When $Q \equiv 2 \bmod 4$, the periodic solution $q(t)$ is a double-choreographic solution. Each closed curve has $\frac{Q}{2}$ sides. The minimum period is $\mathcal{T}=4 Q T$. Body $q_{1}$ chase body $q_{3}$ on a closed curve and body $q_{2}$ chase body $q_{4}$ on another closed curve. $q_{1}(t+2 Q T)=q_{3}(t)$ and $q_{3}(t+2 Q T)=q_{1}(t) \cdot q_{4}(t+2 Q T)=q_{2}(t)$ and $q_{2}(t+2 Q T)=q_{4}(t)$.

- When $Q \equiv 3 \bmod 4$, the periodic solution $q(t)$ is a choreographic solution. The closed curve has $Q$ sides. The minimum period is $\mathcal{T}=8 Q T$. The four bodies chase each other on the closed curve in the order of $q_{1}, q_{4}, q_{3}, q_{2}$, and then $q_{1}$, i.e. $q_{1}(t+2 Q T)=q_{4}(t)$, $q_{4}(t+2 Q T)=q_{3}(t), q_{3}(t+2 Q T)=q_{2}(t)$, and $q_{2}(t+2 Q T)=q_{1}(t)$. 


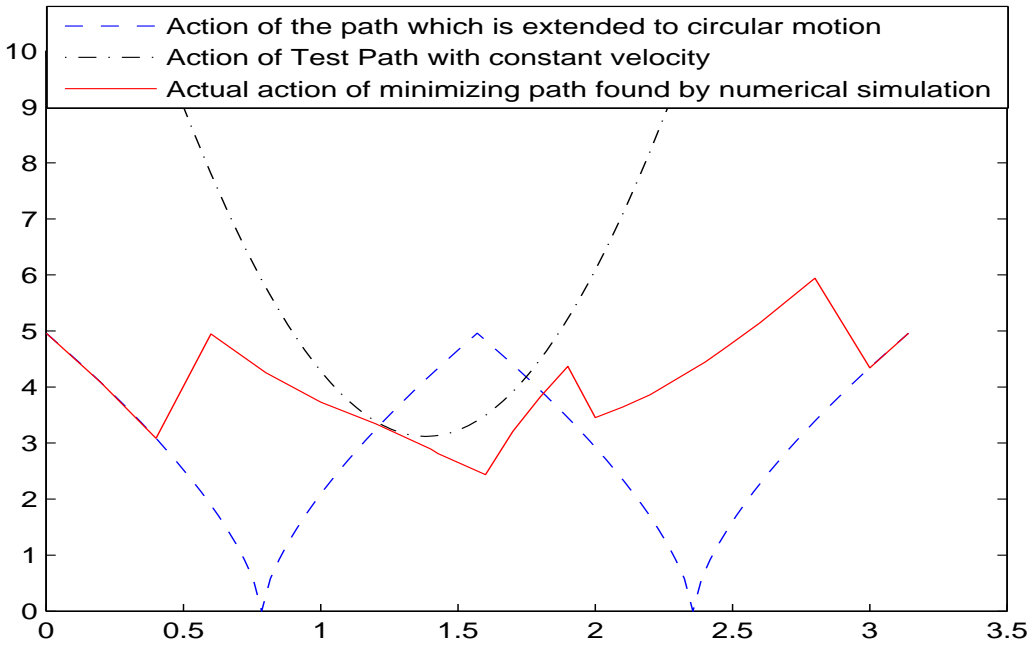

Figure 3: Action levels of test path with constant velocity (dashdotted black), circular motion (dashed blue), and minimizing path (solid red).

(3) [Linear Stability] If $\theta=\frac{P}{2 P+1} \pi$, the simple choreographic solutions $q(t)$ are linearly stable for $P=2,3,4, \cdots, 15$. If $\theta=\frac{2 P-1}{4 P}$, the non-choreographic solutions $q(t)$ are linearly stable for $P=3,4, \cdots, 8$. If $\theta=\frac{2 P-1}{4 P+2}$, the double choreographic solutions $q(t)$ are linearly stable for $P=5,6,7$.

Proof. We first observe that the proposition 2.2 of existence of minimizers in space $\Gamma$ still holds for $\theta \in\left(\theta_{0}, \theta_{1}\right)$ and $\theta \neq \frac{\pi}{2}$ by remark 2.7. The proof of the non-collision theorem 2.3 does not depend on $\theta$ and the extension property (11) in theorem 2.5 is also independent of the rotation angle $\theta$. Now for given $\theta$, we compare the action of the test path $\bar{q}(t)$ with constant velocity for $\vec{a}=[1.0597,1.7696,0.8094,0.7536,1.1032,2.4398]$ and the action of the path $q^{\circ}(t)$ for $\vec{a}^{\circ}$ which is extended to a circular motion. The test path is constructed by connecting $q(0)$ and $q(T)$ by straight line segment with constant velocity. Both actions $\mathcal{A}\left(q^{\circ}(t)\right)$ and $\mathcal{A}(\bar{q}(t))$ are explicit continuous functions of $\theta$ given by formula (38) and (39) respectively (see dashed line and dashdotted line in Figure 3). Both calculations of the functions are provided in appendix $\mathrm{A}$ and appendix B. So there exist $\theta_{0}$ and $\theta_{1}$ such that for $\theta \in\left(\theta_{0}, \theta_{1}\right)$, the action of the test path is smaller than the action of the path $q^{\circ}(t)$. Numerically, $1.1938<\theta_{0}<1.2252$ or $0.38 \pi<\theta_{0}<0.39 \pi$, and $1.7279<\theta_{1}<1.7593$ or $0.55 \pi<\theta_{1}<0.56 \pi$. Therefore there exists a local minimizer with smaller action and the corresponding minimizing path $q^{*}(t)$ on $[0, T]$ can be extended to a non-circular solution $q(t)$ as in theorem 2.5 and

$$
q(t)=\sigma^{k}(q(t-2 k T)) R(2 k \theta) \text { for } t \in(2 k T,(2 k+2) T] \text { and } k \in \mathbf{Z}^{+} .
$$

(1) By the extension formula, it is easy to show that $q(t)$ is a quasi-periodic solution if $\theta$ is not commensurable with $\pi$. See figure 5 .

(2) If $\theta$ is commensurable with $\pi$ and $\theta=\frac{P}{Q} \pi$ where the positive integers $P$ and $Q$ are relatively prime, then $q(t)=\sigma^{4 Q}(q(t-8 Q T)) R(2 P \pi)=q(t-8 Q T)$ which implies that $q(t)$ is a periodic solution. For $0 \leq k<Q$, the trajectory sets $\left\{q_{i}(t) \mid t \in(2 k,(2 k+2) T)\right\} i=1,2,3$, and 4 are all different since the rotation matrix $R\left(2 k \frac{P}{Q} \pi\right)$ is not identity matrix. The four trajectories on which the four body travel in $t \in(0,2 Q T)$ are all different. 
- When $Q \equiv 0 \bmod 4, \sigma^{Q}=\sigma^{0}=[1,2,3,4]$ and $q(t)=q(t-2 Q T)$. So the four different trajectories in $t \in(0,2 Q T)$ are closed on their own at $t=2 Q T$, i.e. $q(2 Q T)=q(0)$. The periodic solution is non-choreographic and the minimum period is $\mathcal{T}=2 Q T$. Each close curve has $\frac{2 Q T}{8 T}=\frac{Q}{4}$ sides. In particular, when $P=1$ and $Q=4$, each closed curve is circle-like (one side); when $P=3$ and $Q=8$, each closed curve is ellipse-like (two sides); when $P=5$ and $Q=12$, each closed curve is triangle-like (three sides); and so on. See figure 6 .

- When $Q \equiv 1 \bmod 4, \sigma^{Q}=\sigma^{1}=[2,3,4,1]$ and $q(t)=\sigma(q(t-2 Q T))$. The four different trajectories in $t \in(0,2 Q T)$ are connected at $t=2 Q T$ as $q_{1}(2 Q T)=q_{2}(0), q_{2}(2 Q T)=$ $q_{3}(0), q_{3}(2 Q T)=q_{4}(0)$, and $q_{4}(2 Q T)=q_{1}(0)$, and they form a closed orbit. Since $\sigma^{4 Q}=[1,2,3,4]$ and $q(t)=q(t-8 Q T), q(t)$ is a simple choreographic solution with minimum period $\mathcal{T}=8 Q T$ and it has $\frac{8 Q T}{8 T}=Q$ sides. In particular, when $Q=5$ and $P=2$, the orbit is a star pentagon (five sides) (See figure 1). When $Q=9$ and $P=4$, the orbit is a star nonagon (nine sides). When $Q=13$ and $P=6$, the orbit is a star tridecagon (thirteen sides); and so on. See figure 7.

- When $Q \equiv 2 \bmod 4, \sigma^{Q}=\sigma^{2}=[3,4,1,2]$ and $q(t)=\sigma^{2}(q(t-2 Q T))$. The four different trajectories in $t \in(0,2 Q T)$ are connected at $t=2 Q T$ in two pairs, i.e. $q_{1}(2 Q T)=q_{3}(0)$ and $q_{3}(2 Q T)=q_{1}(0), q_{2}(2 Q T)=q_{4}(0)$ and $q_{4}(2 Q T)=q_{2}(0)$. So they form two closed orbits. Since $\sigma^{2 Q}=[1,2,3,4]$ and $q(t)=q(t-4 Q T), q(t)$ is a double-choreographic solution with minimum period $\mathcal{T}=4 Q T$ and each closed orbit has $\frac{4 Q T}{8 T}=\frac{Q}{2}$ sides. In particular, when $Q=10$ and $P=3$, the orbit is the combination of two flowers with five petals each; and so on. See figure 8

- When $Q \equiv 3 \bmod 4, \sigma^{Q}=\sigma^{3}=[4,1,2,3]$ and $q(t)=\sigma^{3}(q(t-2 Q T))$. The four different trajectories in $t \in(0,2 Q T)$ are connected at $t=2 Q T$ as $q_{1}(2 Q T)=q_{4}(0)$, $q_{4}(2 Q T)=q_{3}(0), q_{3}(2 Q T)=q_{2}(0)$, and $q_{2}(2 Q T)=q_{1}(0)$, and they form a closed orbit. Since $\sigma^{4 Q}=[1,2,3,4]$ and $q(t)=q(t-8 Q T), q(t)$ is a simple choreographic solution with minimum period $\mathcal{T}=8 Q T$ and it has $\frac{8 Q T}{8 T}=Q$ sides. In particular, when $Q=7$ and $P=3$, the orbit is a star heptagon (seven sides); and so on. See figure 9 .

(3) We prove the stability for these cases as what we have done for star pentagon. We first numerically compute the Monodromy matrix $X(\mathcal{T})$ of the reduced Hamiltonian system $H_{4}$ as in section 4 . Then we compute $W=\frac{1}{2}\left(X+X^{-1}\right)$ and its eigenvalues. To check whether the global error is within the expected accuracy, we also compute the monodromy matrix and its eigenvalues with several different step sizes for each case. Here we only list the eigenvalues for a few cases and their initial conditions are given in appendix C. The four pairs of eigenvalues are real and distinct in $(-1,1)$. Returning to the full monodromy matrix, the corresponding eigenvalues are distinct and on the unit circle. Therefore, the corresponding periodic solutions are all linearly stable.

$\theta=\frac{3 \pi}{7}, \mathcal{T}=56$, the four pairs of eigenvalues are [-0.375476, 0.493924, 0.623185, 0.698755].

$\theta=\frac{4 \pi}{9}, \mathcal{T}=72$, the four pairs of eigenvalues are [ $\left.-0.888315,0.717492,0.781167,0.875241\right]$.

$\theta=\frac{5 \pi}{12}, \mathcal{T}=24$, the four pairs of eigenvalues are [ $\left.-0.752385,0.786314,0.850377,0.845072\right]$.

$\theta=\frac{9 \pi}{22}, \mathcal{T}=88$, the four pairs of eigenvalues are $[-0.612649,-0.791503,-0.967491$, $-0.99911]$. 
Remark 5.2. What we want to point out here is that the test path used in Figure 3 is generated by a fixed SPBC. There exist local minimizers for $\theta$ out of the interval $\left[\theta_{0}, \theta_{1}\right]$ since the action of the test path may be not good for certain angle $\theta$. There also exist local minimizers which have higher actions than their circular solutions. When $\theta$ is small (near zero) or large (near $\pi$ ), it is hard to numerically find minimizers for non-circular motion. In figure 3, some actual actions of minimizing pathes are numerically found for $\theta \in[0.2,3.2]$. But the actual action of minimizing path is not used in the proof of theorem 5.1 and it is enclosed here only for the purpose of comparison.

\section{Appendix A: Action of the path which is extended to a circular solution}

The configuration $q$ is called a central configuration if $q$ satisfies the following nonlinear algebraic equation system:

$$
\lambda\left(q_{i}-c\right)-\sum_{j=1, j \neq i}^{n} \frac{m_{j}\left(q_{i}-q_{j}\right)}{\left|q_{i}-q_{j}\right|^{3}}=0, \quad 1 \leq i \leq n,
$$

for a constant $\lambda$, where $c=\left(\sum m_{i} q_{i}\right) / M$ is the center of mass and $M=m_{1}+m_{2}+\cdots+m_{n}$ is the total mass. We recall the fact that coplanar central configurations always admit homographic solutions where each body executes a similar Keplerian ellipse of eccentricity $e, 0 \leq e \leq 1$. When $e=0$, the relative equilibrium solutions are consisting of uniform circular motion for each of the masses about the common center of mass. When $e=1$, the homographic solutions degenerate to a homothetic solution which includes total collision, together with a symmetric segment of ejection. Gordon found that for fixed period $\mathcal{T}^{\circ}$, all of the homographic solutions have the same action ([18]). Consider the circular solution of the four-body problem with equal masses

$$
q_{k}^{\circ}(t)=r\left(\cos \left(\omega t+\rho_{k}\right), \sin \left(\omega t+\rho_{k}\right)\right), k=1,2,3,4,
$$

where $r>0$ is the radius of the circle and $\rho_{k}=\frac{k \pi}{2}$. We can easily find the relation between $\omega$ and $r$ by Newtonian equations (2):

$$
r^{3} \omega^{2}=\frac{1}{4} U_{0}
$$

where $U_{0}=2 \sqrt{2}+1$ is the potential energy of the four equal masses at the square configuration on a unit circle. The minimum period is $\mathcal{T}^{\circ}=\frac{2 \pi}{\omega}$. The minimum value of the action functional (1) (realized by the circular solution) could be computed

$$
\begin{gathered}
\mathcal{A}(q(t))=\int_{0}^{\mathcal{T}^{\circ}} \sum_{k=1}^{4} \frac{1}{2} m_{k}\left|\dot{q}_{k}(t)\right|^{2}+U(q(t)) d t=\left(2 r^{2} \omega^{2}+\frac{U_{0}}{r}\right) \mathcal{T}^{\circ} \\
=\frac{3 U_{0}}{2 r} \mathcal{T}^{\circ}=3(2)^{\frac{1}{3}} U_{0}^{\frac{2}{3}} \pi^{\frac{2}{3}}\left(\mathcal{T}^{\circ}\right)^{\frac{1}{3}} .
\end{gathered}
$$

If the bodies rotate an angle $\alpha$ in the time interval $[0, T], \omega=\frac{\alpha}{T}$ and $\mathcal{T}^{\circ}=\frac{2 \pi T}{\alpha}$ and the action over the time interval $[0, T]$ is

$$
3(2)^{\frac{1}{3}} U_{0}^{\frac{2}{3}} \pi^{\frac{2}{3}}\left(\mathcal{T}^{\circ}\right)^{\frac{1}{3}} \frac{\alpha}{2 \pi}=3(2)^{-\frac{1}{3}} U_{0}^{\frac{2}{3}} T^{\frac{1}{3}} \alpha^{\frac{2}{3}} \approx 5.8271766 T^{\frac{1}{3}} \alpha^{\frac{2}{3}}
$$



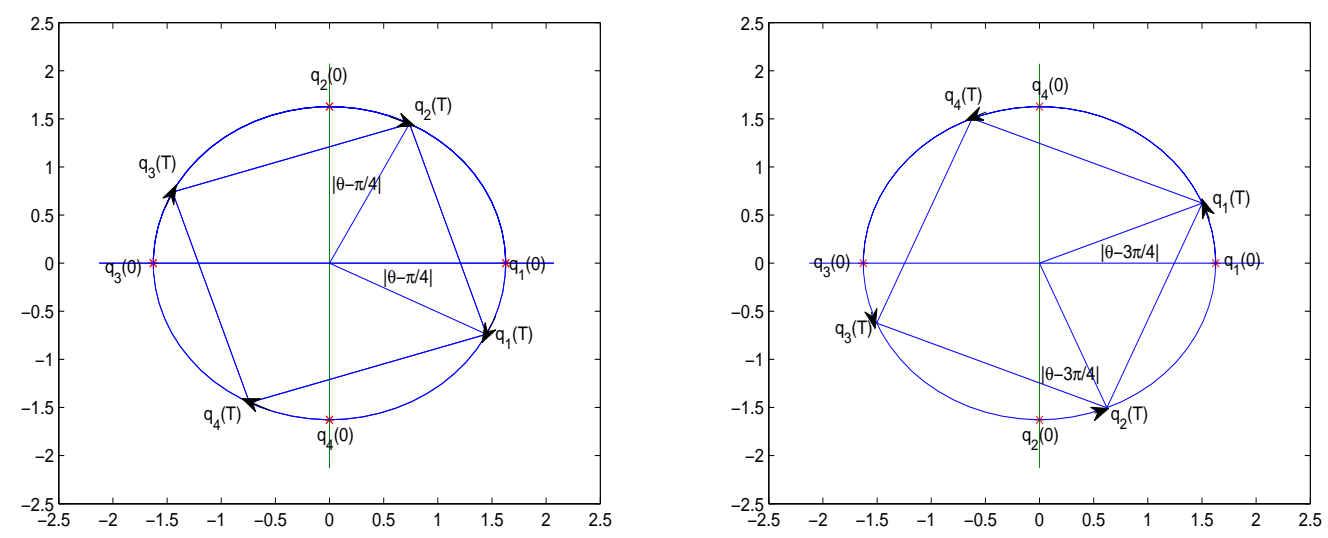

Figure 4: Left: For $\frac{\pi}{4}<\theta<\frac{\pi}{2}, \vec{a}^{\circ}=\left[a_{1}, 0,-a_{1}, \frac{\sqrt{2} a_{1}}{2},-\frac{\sqrt{2} a_{1}}{2}, \frac{\sqrt{2} a_{1}}{2}\right]$ with $a_{1}>0$ and $\alpha=\left|\theta-\frac{\pi}{4}\right|$. Right: For $\frac{\pi}{2}<\theta<\frac{3 \pi}{4}, \vec{a}^{\circ}=\left[a_{1}, 0, a_{1}, \frac{\sqrt{2} a_{1}}{2}, \frac{\sqrt{2} a_{1}}{2},-\frac{\sqrt{2} a_{1}}{2}\right]$ with $a_{1}>0$ and $\alpha=\left|\theta-\frac{3 \pi}{4}\right|$.

For fixed $T>0$, the configurations generating a circular solution should be always a square and the bodies rotate an angle $\alpha$ as small as possible in order to have a minimizing action over the time interval $[0, T]$. By the structure of our prescribed boundary conditions, if $\frac{\pi}{4}<\theta<\frac{\pi}{2}$, the angle that the bodies rotate in the time interval $[0, T]$ is $\alpha=\theta-\frac{\pi}{4}$ (see the left graph of Figure 4). And the SPBC is given by $\vec{a}^{\circ}=\left[a_{1}, 0,-a_{1}, \frac{\sqrt{2} a_{1}}{2},-\frac{\sqrt{2} a_{1}}{2}, \frac{\sqrt{2} a_{1}}{2}\right]$ which generates a circular solution with $r=a_{1}, \omega=\frac{\theta-\frac{\pi}{4}}{T}$. The radius $a_{1}=U_{0}^{1 / 3} T^{2 / 3}(2 \alpha)^{-2 / 3}$ of the circular solution is uniquely determined by $T$ and the corresponding angle. So the exact formula for action $\mathcal{A}_{\vec{a}^{\circ}}\left(q^{\circ}(t)\right)$ of the path $q^{\circ}(t)$ in the time $[0, T]$ which can generate a circular motion with period $\mathcal{T}^{\circ}$ is

$$
\mathcal{A}\left(q^{\circ}(t)\right)=3(2)^{-\frac{1}{3}} U_{0}^{\frac{2}{3}} T^{\frac{1}{3}}\left(\theta-\frac{\pi}{4}\right)^{\frac{2}{3}},
$$

where $\mathcal{T}^{\circ}=\frac{2 \pi T}{\theta-\frac{\pi}{4}}$. For $T=1$ and $\theta=\frac{2 \pi}{5}, a_{1}=1.6272$, and

$$
\mathcal{A}\left(q^{\circ}(t)\right) \approx 3.528734094 \text {. }
$$

If $\frac{\pi}{2}<\theta<\frac{3 \pi}{4}$, the angle that the bodies rotate in the time interval $[0, T]$ is $\alpha=\left|\theta-\frac{3 \pi}{4}\right|$ (see the right graph of Figure 4). And the SPBC is given by $\vec{a}^{\circ}=\left[a_{1}, 0, a_{1}, \frac{\sqrt{2} a_{1}}{2}, \frac{\sqrt{2} a_{1}}{2},-\frac{\sqrt{2} a_{1}}{2}\right]$. For other $\theta$, we have similar results. If $\frac{3 \pi}{4}<\theta<\pi, \alpha=\left|\theta-\frac{3 \pi}{4}\right|$. If $0<\theta<\frac{\pi}{4}, \alpha=\left|\theta-\frac{\pi}{4}\right|$.

\section{Appendix B: Action of Test Path with Constant Velocity}

Given $\theta, T$ and $\vec{a}=\left(a_{1}, a_{2}, \cdots, a_{6}\right)$, the test path $\bar{q}(t)$ with constant velocity connecting the structural prescribed boundary conditions is given by

$$
\begin{gathered}
\bar{q}(t)=Q s t a r t+\frac{t(\text { Qend }- \text { Qstart })}{T}, t \in[0, T] . \\
\mathcal{A}(\bar{q}(t))=\sum_{k=1}^{4} \frac{1}{2 T} m_{k} \mid \text { Qend }_{k}-\text { Qstart }\left._{k}\right|^{2} \\
+\int_{0}^{T} \sum_{1 \leq k<j \leq 4} \frac{m_{k} m_{j}}{\mid\left(\text { Qstart }_{k}-\text { Qstart }_{j}\right)\left(1-\frac{t}{T}\right)+\left(\text { Qend }_{k}-\text { Qend }_{j}\right) \frac{t}{T} \mid} d t
\end{gathered}
$$


where the integrals only involve the form of $\int_{0}^{T} \frac{m_{k} m_{j}}{\left(a+b t+c t^{2}\right)^{1 / 2}} d t$ which can be integrated explicitly by trigonometric substitution. In figure 3 , the action of test path is computed by assuming $\vec{a}=$ $[1.0597,1.7696,0.8094,0.7536,1.1032,2.4398]$ with $T=1$ and the action is an explicit function of $\theta$. For example, for $T=1$ and $\theta=\frac{2 \pi}{5}, \mathcal{A}(\bar{q}(t))=1.0633+\int_{0}^{1} \frac{1}{\left(7.7742-6.1698 * t+3.2638 * t^{2}\right)^{1 / 2}}+$ $\cdots+\frac{1}{\left(21.3675+2.22208 * t+.2208 * t^{2}\right)^{1 / 2}}=3.2484$.

\section{Appendix C: Numerical simulations for the orbits with different rotation angle $\theta$.}

Here we present some numerical simulations for the orbits with different rotation angle $\theta$. Our searching Matlab program consists two parts. The first part is to find a solution satisfying the boundary conditions of SPBC for a given $\vec{a}=\left(a_{1}, a_{2}, \cdots, a_{6}\right)$ and return its action $\mathcal{A}$. The existence of such solution is guaranteed by Lemma 2.1. The second part is to find a local minimizer by varying the parameters $a_{1}, a_{2}, \cdots, a_{6}$. The existence of such local minimizer is shown in proposition 2.2. We would like to mention the following: (A) Local minimizers are not unique. (B) There are two shapes of orbits: convex (such as star pentagon) and concave (such as those orbits in Figure 12). (C) In numerical searching, local minimizers depend on an initial guess in general.

All the orbits presented in the paper are extended from the initial four pieces connecting from $q(0)$ to $q(T)$ by extension formula (11). Most of the figures can be generated in any Newtonian $n$-body simulation program by using these initial data. However, as we point out in Remark 1.4, non-circular minimizers exist for $\theta$ out of the interval $\left[\theta_{0}, \theta_{1}\right]$ and those solutions are numerically unstable. Some unstable solutions even can not run a full period in simulation and it is hard to produce satisfactory numerical figures. Sometimes we have to refine our searching by providing a better guess. We list the initial conditions for some stable orbits in our figures.

$$
\begin{gathered}
\theta=\frac{3 \pi}{7}, q_{1}(0)=[0.9421633223,2.189470662], \dot{q}_{1}(0)=[-0.4908748479,-0.474846875], \\
q_{2}(0)=[0,-1.300537268], \dot{q}_{2}(0)=[1.039561987,0], q_{3}(0)=[-0.9421633223,2.189470662], \\
\dot{q}_{3}(0)=[-0.4908732773,0.4748477727], q_{4}(0)=[0,-3.078404055], \dot{q}_{4}(0)=[-0.05781386229,0] . \\
\theta=\frac{4 \pi}{9}, q_{1}(0)=[0.886183635,2.574192004], \dot{q}_{1}(0)=[-0.4491532002,-0.5077584354], \\
q_{2}(0)=[0,-1.721056291], \dot{q}_{2}(0)=[1.002413363,0], q_{3}(0)=[-0.886183635,2.574192004], \\
\dot{q}_{3}(0)=[-0.4491265528,0.5077539042], q_{4}(0)=[0,-3.427327717], \dot{q}_{4}(0)=[-0.1041336098,0] . \\
\theta=\frac{5 \pi}{12}, q_{1}(0)=[0.9885667998,1.984831768], \dot{q}_{1}(0)=[-0.5187341985,-0.4460463326], \\
q_{2}(0)=[0,-1.067317853], \dot{q}_{2}(0)=[1.064058961,0], q_{3}(0)=[-0.9885667998,1.984831768], \\
\dot{q}_{3}(0)=[-0.5187107584,0.4460354291], q_{4}(0)=[0,-2.902345684], \dot{q}_{4}(0)=[-0.02661400412,0] . \\
\theta=\frac{9 \pi}{22}, q_{1}(0)=[1.020078100,1.878808307], \dot{q}_{1}(0)=[-0.5352327448,-0.4256795762], \\
q_{2}(0)=[0,-0.9422097516], \dot{q}_{2}(0)=[1.078223783,0], q_{3}(0)=[-1.02007810,1.878808307], \\
\dot{q}_{3}(0)=[-0.5352124256,0.4256692972], q_{4}(0)=[0,-2.815406862], \dot{q}_{4}(0)=[-0.007778613051,0] .
\end{gathered}
$$

\section{Acknowledgements}

This work was partially supported by a Mathematician Collaboration Grant from the Simons Foundation (\#278445 to Zhifu Xie) and a grant from NSF (HRD-1409939 to Zhifu Xie). 

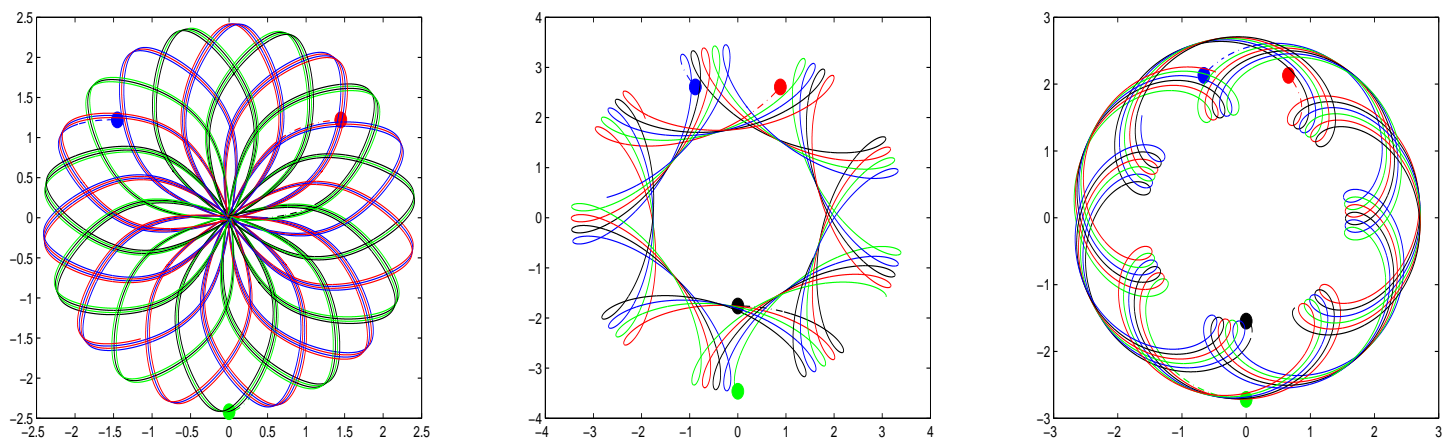

Figure 5: Quasi-Periodic Solutions on $[0,80 T]$. From left to right $\theta=1.0,1.4,1.8$.
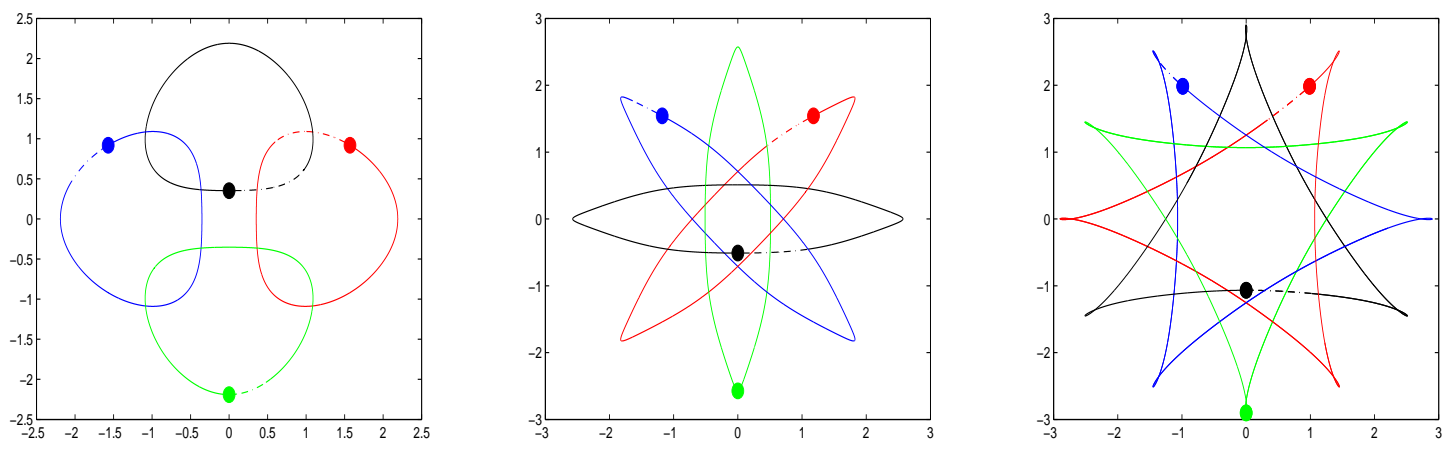

Figure 6: Non-Choreographic Periodic Solutions for $\theta=\frac{P}{Q} \pi$ with $Q \equiv 0 \bmod 4$. From left to right $\theta=\frac{\pi}{4}, \frac{3 \pi}{8}$, and $\frac{5 \pi}{12}$.
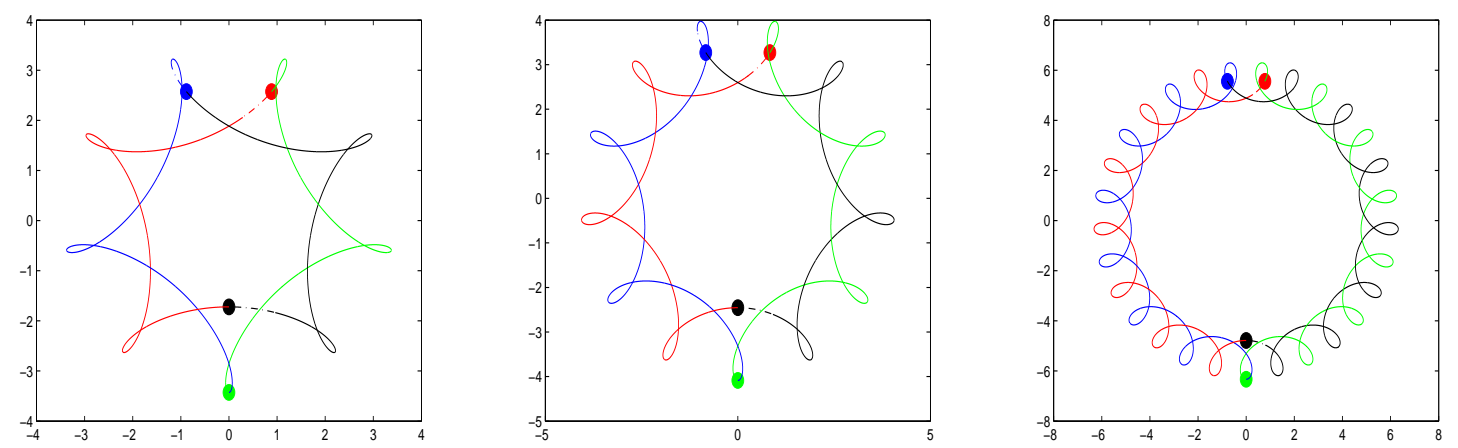

Figure 7: Simple Choreographic Solutions for $\theta=\frac{P}{Q} \pi$ with $Q \equiv 1 \bmod 4$. Bodies chase each other in the order $q_{1}($ red $) \rightarrow q_{2}($ black $) \rightarrow q_{3}($ blue $) \rightarrow q_{4}$ (green $) \rightarrow q_{1}$. From left to right $\theta=\frac{4 \pi}{9}, \frac{6 \pi}{13}$, and $\frac{14 \pi}{29}$. 

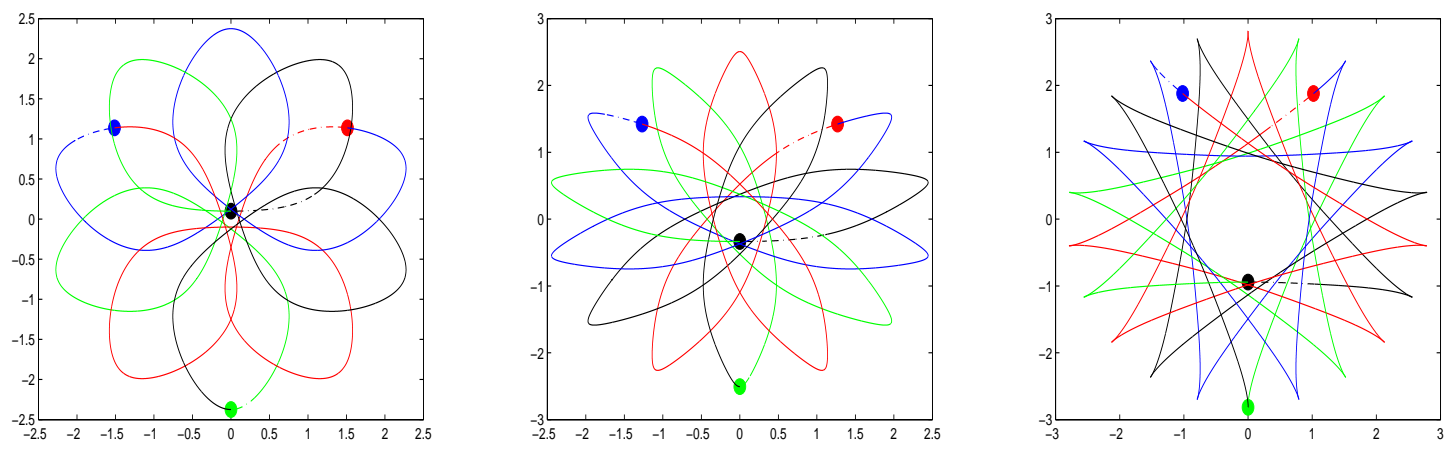

Figure 8: Double-Choreographic Solutions for $\theta=\frac{P}{Q} \pi$ with $Q \equiv 2 \bmod 4$. Bodies chase each other pairwisely as $q_{1}($ red $) \rightarrow q_{3}($ blue $) \rightarrow q_{1}$ and $q_{2}($ black $) \rightarrow q_{4}($ green $) \rightarrow q_{2}$. From left to right $\theta=\frac{3 \pi}{10}, \frac{5 \pi}{14}$, and $\frac{9 \pi}{22}$.
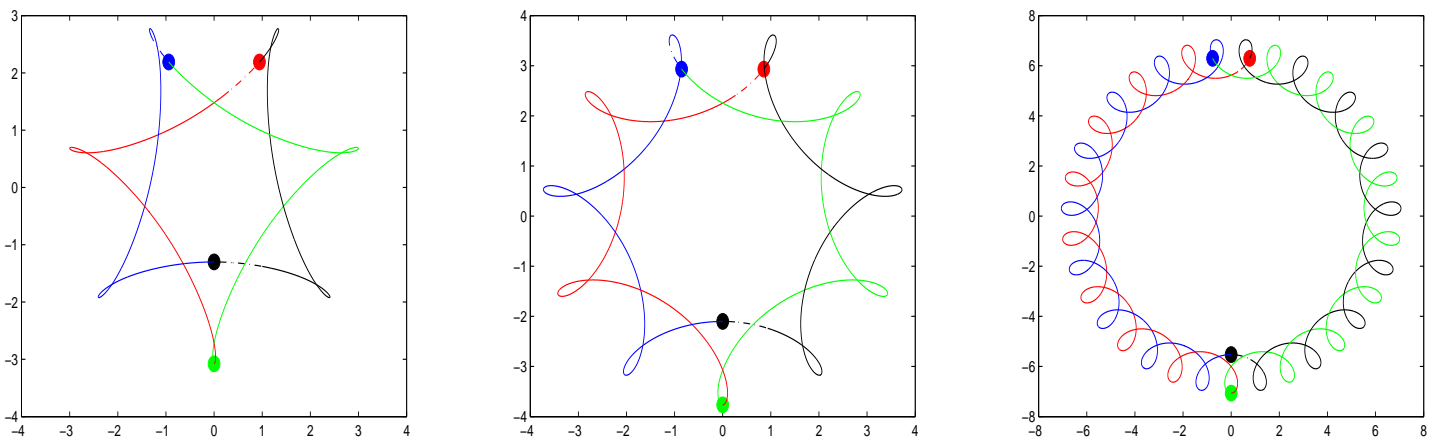

Figure 9: Simple Choreographic Solutions for $\theta=\frac{P}{Q} \pi$ with $Q \equiv 3 \bmod 4$. Bodies chase each other in the order $q_{1}($ red $) \rightarrow q_{4}($ green $) \rightarrow q_{3}($ blue $) \rightarrow q_{2}($ black $) \rightarrow q_{1}$. From left to right $\theta=\frac{3 \pi}{7}, \frac{5 \pi}{11}$, and $\frac{17 \pi}{35}$.
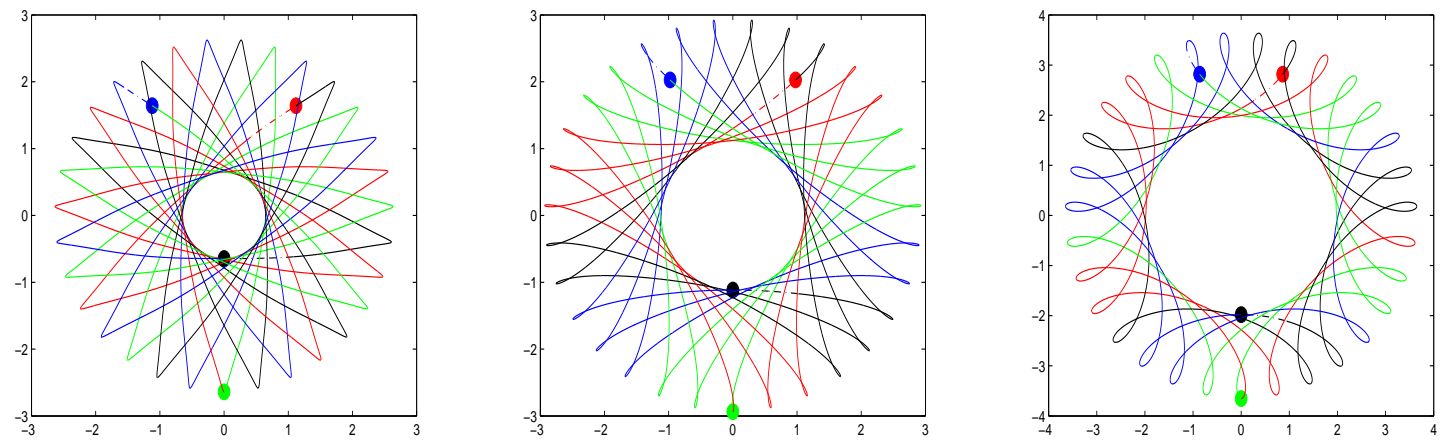

Figure 10: Periodic solutions with same periods $\mathcal{T}=248 T$. From left to right $\theta=\frac{12 \pi}{31}, \frac{13 \pi}{31}$, and $\frac{14 \pi}{31}$. 

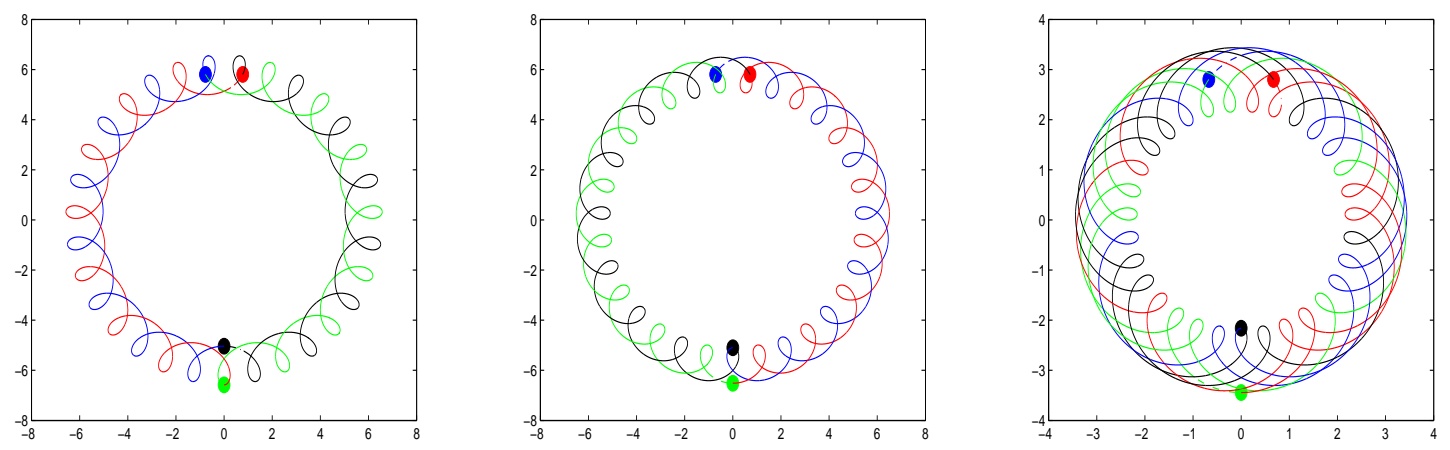

Figure 11: Periodic solutions with same periods $\mathcal{T}=248 T$. From left to right $\theta=\frac{15 \pi}{31}, \frac{16 \pi}{31}$, and $\frac{17 \pi}{31}$.
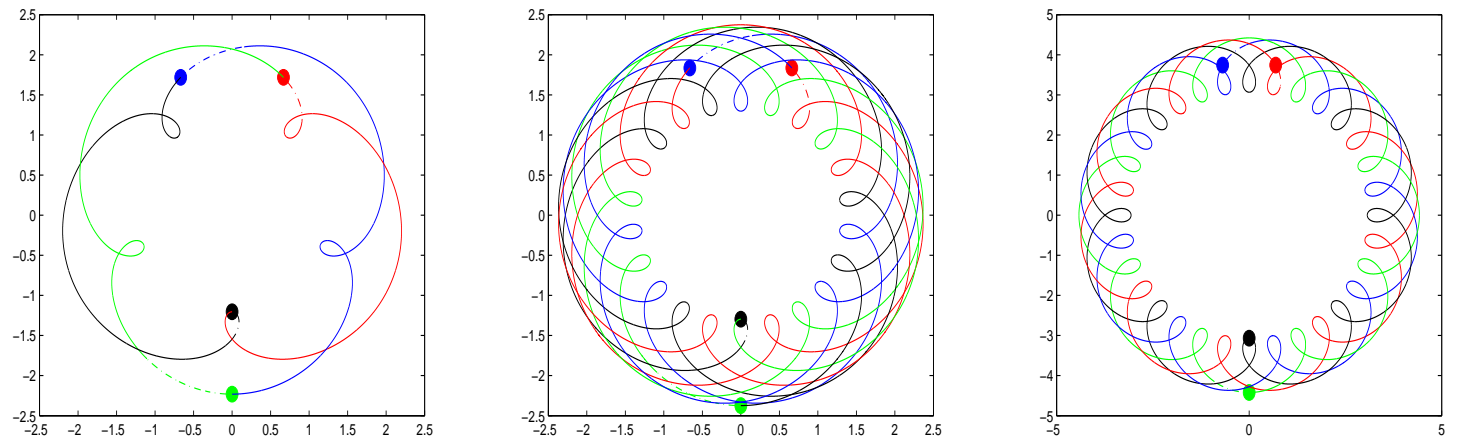

Figure 12: Periodic solutions for $\theta>\frac{\pi}{2}$. From left to right $\theta=\frac{3 \pi}{5}, \frac{13 \pi}{22}$, and $\frac{17 \pi}{32}$. 


\section{References}

[1] G. Arioli, V. Barutello, S. Terracini, A new branch of Mountain Pass solutions for the choreographical 3-body problem. Comm. Math. Phys. 268 (2006), no. 2, 439-463.

[2] V. Barutello, S. Terracini, Double choreographical solutions for n-body type problems, Celestial Mech. Dynam. Astronom. 95 (2006) 67-80.

[3] V. Barutello, S. Terracini, Action minimizing orbits in the n-body problem with simple choreography constraint. Nonlinearity 17 (2004), no. 6, 2015-2039.

[4] E. Barrabs, J. Cors, C. Pinyol, J. Soler, Hip-hop solutions of the 2N-body problem. Celestial Mech. Dynam. Astronom. 95 (2006), no. 1-4, 55-66.

[5] R. Broucke, Classification of Periodic Orbits in the Four- and Five-Body Problems, Ann. N.Y. Acad. Sci. 1017 (2004), 408421.

[6] A. Chenciner, Action minimizing solutions in the Newtonian n-body problem: from homology to symmetry. Proceedings of the International Congress of Mathematicians (Beijing, 2002). Higher Ed. Press, Beijing, 279-294, 2002. Erratum. Proceedings of the International Congress of Mathematicians (Beijing, 2002). Higher Ed. Press, Beijing, (2002), 651-653.

[7] A. Chenciner, J. Fejoz, Unchained polygons and the $N$-body problem, Regular and chaotic dynamics, Vol. 14 (2009), No 1, 64-115 .

[8] A. Chenciner, R. Montgomery, A remarkable periodic solution of the three body problem in the case of equal masses. Ann. Math. 152 (2000), 881-901.

[9] A. Chenciner, J. Gerver, R. Montgomery, C. Simó, Simple choreographic motions of $N$ bodies: a preliminary study. Geometry, mechanics, and dynamics, 287-308, Springer, New York, 2002.

[10] A. Chenciner, A. Venturelli, Minima of the action integral of the Newtonian problem of four bodies of equal mass in $R^{3}$ : "hip-hop" orbits, Celestial Mech. Dynam. Astronom. 77 (2000), no. 2, 139-152.

[11] K. Chen, Existence and minimizing properties of retrograde orbits to the three-body problem with various choices of masses. Ann. of Math. 167 (2008), no. 2, 325-348.

[12] K. Chen, Variational methods on periodic and quasi-periodic solutions for the N-body problem. Ergodic Theory Dynam. Systems 23 (2003), no. 6, 1691-1715.

[13] K. Chen, T. Ouyang, Z. Xia, Action-minimizing periodic and quasi-periodic solutions in the $N$-body problem, Math. Res. Lett. 19 (2012), no. 2, 483-497.

[14] C. Deng, S. Zhang, Q. Zhou, Rose solutions with three petals for planar 4-body problems, Sci. China Math, 2010, 53(12): 3085-3094.

[15] C. Deng, S. Zhang, New periodic solutions for planar $N+2$-body problems. J. Geom. Phys. 61 (2011), no. 12, 2369-2377.

[16] G. Fusco, G.F. Gronchi, P. Negrini, Platonic polyhedra, topological constraints and periodic solutions of the classical N-body problem, Invent. math. 185 (2011), 283-332. 
[17] D. Ferrario, S. Terracini, On the existence of collisionless equivariant minimizers for the classical n-body problem, Invent. math. 155 (2004), 305-362.

[18] W. Gordon, A minimizing property of Keplerian orbits, American Journal of Mathematics 99, no. 5, (1977) 961-971.

[19] X. Hu, Y. Long, and S. Sun, Linear stability of elliptic Lagrangian solutions of the classical planar three-body problem via index theory, Arch. Rational Mech. Anal. 213 (2014) 9931045.

[20] X. Hu and S. Sun, Index and Stability of Symmetric Periodic Orbits in Hamiltonian Systems with Application to Figure-Eight Orbit, Commun. Math. Whys., vol. 290 (2009), no. 2, pp. 737-777.

[21] T. Kapela, C. Simó, Computer assisted proofs for nonsymmetric planar choreographies and for stability of the Eight, Nonlinearity, 20 (2007), 1241-1255.

[22] Y. Long, $\omega$-index theory and linear stability of elliptic Lagrangian solutions of the classical three-body problem. Adv. Nonlinear Stud. 12 (2012), no. 4, 799-818.

[23] C. Marchal, How the method of minimization of action avoids singularities, Celestial Mech. Dynam. Astronom., 83 (2002) 325-353.

[24] C. Moore, Braids in Classical Gravity, Physical Review Letters 70 (1993) 3675-3679.

[25] D. Offin, H. Cabral, Hyperbolicity for symmetric periodic orbits in the isosceles three body problem. Discrete Contin. Dyn. Syst. Ser. S 2 (2009), no. 2, 379-392.

[26] T. Ouyang, Z. Xie, A continuum of periodic solutions to the planar four-body problem with various choices of masses, submitted.

[27] T. Ouyang, D. Yan, Existence of spatial isosceles periodic orbits in three-body problem, in progress.

[28] H. Poincaré, Sur les solutions périodiques et le principe de moindre action, C.R. Acad, Sci, Paris. 123 (1896), 915-918.

[29] K. Meyer, G. Hall, D. Offin, Introduction to Hamiltonian Dynamical Systems and the N-Body Problem, second edition, Springer, 2009.

[30] G. Roberts, Linear stability ananlysis of the figure-eight orbit in the three-body problem, Ergod. Th. and Dynam. Sys. (2007), 27, 1947-1963.

[31] D. Saari, The manifold structure for collision and for hyperbolic-parabolic orbits in the n-body problem, J. Differential Equations, 55 (1984), 300-329.

[32] C. Simó, New families of solutions in the $N$-body problems, Proceedings of the third European Congress of Matheamtics, Casacuberta et al. edits, Progress in Mathematics (2001), 201, 101-115.

[33] H.J. Sperling, On the real singularities of the $N$-body problem, J. Reine Angew. Math. $245,(1970), 15-40$.

[34] K.F. Sundman, Mémoire sur le problèdes trois corps. Acta Math. 36, (1913), 105-179. 
[35] S. Terracini, On the variational approach to the periodic $n$-body problem, Celestial Mech. Dynam. Astronom. 95 (2006), 1-4, 3-25.

[36] S. Terracini, A. Venturelli, Symmetric trajectories for the $2 N$-body problem with equal masses, Arch. Rational Mech. Anal. 184 (2007), 465-493.

[37] R.J. Vanderbei, New Orbits for the n-Body Problem. In Proceedings of the Conference on New Trends in Astrodynamics, 2003.

[38] S. Zhang, Q. Zhou, Variational methods for the choreography solution to the three-body problem. Sci. China Ser. A 45 (2002), no. 5, 594-597 